Article

\title{
On Designing Non-Parametric EWMA Sign Chart under Ranked Set Sampling Scheme with Application to Industrial Process
}

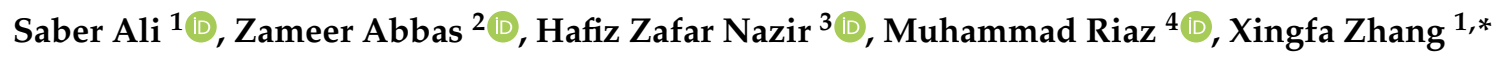 \\ and Yuan $\mathrm{Li}^{1}$ \\ 1 School of Economics and Statistics, Guangzhou University, Guangzhou 510000, China; \\ saber@e.gzhu.edu.cn (S.A.); mathly@gzhu.edu.cn (Y.L.) \\ 2 Department of Statistics, Government Ambala Muslim College Sargodha, Sargodha 40100, Pakistan; \\ zameerstats@gmail.com \\ 3 Departemnt of Statistics, University of Sargodha, Sargodha 40100, Pakistan; hafizzafarnazir@yahoo.com \\ 4 Department of Mathematics and Statistics, King Fahad University of Petroleum and Minerals, \\ Dhahran 31261, Saudi Arabia; riaz76qau@yahoo.com \\ * Correspondence: xingfazhang@gzhu.edu.cn
}

Received: 30 July 2020; Accepted: 1 September 2020; Published: 4 September 2020

\begin{abstract}
Statistical process control (SPC) tools are used for the investigation and identification of unnatural variations in the manufacturing, industrial, and service processes. The control chart, the basic and the most famous tool of SPC, is used for process monitoring. Generally, control charts are constructed under normality assumption of the quality characteristic of interest, but in practice, it is quite hard to hold the normality assumption. In such situations, parametric charts tend to offer more frequent false alarms and invalid out-of-control performance. To rectify these problems, non-parametric control charts are used, as these have the same in-control run length properties for all the continuous distributions and are known as in-control robust. This study intends to develop a new non-parametric exponentially weighted moving average (NPEWMA) chart based on sign statistics under a ranked set sampling scheme that is hereafter named (NPREWMA-SN). The run-length profiles of the NPREWMA-SN chart are computed using the Monte Carlo simulation method. The proposed scheme is compared with NPEWMA-SN and classical EWMA- $\bar{X}$ charts, using different run length measures. The comparison reveals the in-control robustness and superiority of the proposed scheme over its competitors in detecting all kinds of shifts in the process location. A practical application related to the substrate manufacturing process is included to show the demonstration of the proposed chart.
\end{abstract}

Keywords: average run length; control chart; sign test; in-control robustness; manufacturing process; non-parametric; ranked set scheme

\section{Introduction}

Quality management provides many operational and management techniques that save both time and cost to achieve the standard finished product. These techniques are used in manufacturing processes, filling processes, and in services to spot the un-natural variations that improve the quality of finished products. A process can be defined as "systematic way of a number of inputs and a required output" (cf. [1]). For example, in semiconductor manufacturing processes, the controllable input variables are photolithography, temperature, silicon wafer, resistance, and some other process variables. The flow width of the resistance is a running process that has some quality characteristics. These quality characteristics are monitored using various statistical tools to improve and produce 
quality products. For this purpose, statistical process control (SPC) plays an important role. SPC tools are frequently used in the fields of industries, natural sciences, environmental sciences, medicines, and services to monitor the processes. A control chart has a vital role in SPC that is often used in process monitoring to identify any nonconformity in the process parameter. Variation is an unavoidable part of every process output that reduces quality. Common causes of variation do not affect the conformance of the process, while special causes of variation influence the process outputs (cf. [1]). Control charts help us in the early detection of assignable variations that reduce the financial penalties. These abilities of SPC are helpful in producing the quality products in the aforementioned fields.

The concept of control chart, to improve the quality of the process output, was first initiated by Walter A. Shewhart in the early 1920s. Since then, the control chart has a vital role in modern SPC. The Shewhart control charts proposed by [2] are frequently used where large changes occur in process target(s). However, these charts are unable to spot the small and persistent shifts in the process location sharply. Today, advance process monitoring schemes include the cumulative sum (CUSUM) and the exponentially weighted moving average (EWMA) control charts. These are memory-type control charts, as their charting structures are a combination of current and past information in the sample. Although their performance toward small and moderate shift detection is almost same, but most of the quality practitioners prefer the EWMA chart due to its simplicity. The use of these control charts is increasing due to their sensitive nature toward sustained changes in the process parameters. For this purpose, these are regularly used to spot small changes in the process location and scale parameter(s), where small changes may cause serious quality issues.

In practice, it is assumed that the quality characteristic of interest has some distributional assumptions, usually normality assumptions. In real situations, we negate this assumption, and so the usual parametric control charts cannot be implemented with certainty, as these produce more false alarms and invalid comparison. In modern process monitoring schemes, non-parametric (NP) control charts (or distribution-free charts) are a more robust alternative to the parametric charts when the actual process distribution is doubtful. The main advantages of these NP charts is that they do not have any distributional assumption (i.e., normality assumption), while their in-control (IC) run length (RL) distribution remains constant for all the continuous process distributions (cf. [3]). This characteristic of NP charts provides full knowledge and stability of IC and out-of-control (OOC) properties that are compulsory to implement a control chart. According to these limitations, a number of studies were proposed on NP design structures for process monitoring. Some of the studies have used sign (SN) and signed rank (SR) tests, which are the simplest ones under the NP structure (cf. [4]). Using a SN test, Ref. [5] proposed an NP Shewhart-type chart for monitoring the process target. A distribution-free Shewhart chart based on an SR test was proposed by [6] using grouped observations to monitor the process mean. These Shewhart-type charts under NP structure were also discussed in [7] and provided sufficient knowledge about their IC RL properties. A phase-II Shewhart-type NP chart based on Mann-Whitney statistic, for monitoring the process target, was proposed by [8]. Using runs rules, two NP Shewhart-type control charts were proposed by [9] for monitoring the unknown process location. However, these charts efficiently performed for large shifts only and failed to detect small and persistent shifts in the process target. The SN and SR tests were also used in a CUSUM control chart and have shown better performance than the Shewhart-type control chart to monitor the process location (cf. [10]). An NPCUSUM chart based on exceedance statistic was proposed by [11] for monitoring the process location. The only drawback of the CUSUM chart is that it involves very complicated calculations making it difficult to use, whereas the EWMA charts have the same level of performance with a simple design structure and are easy to apply in practical scenarios. For more details on NP control charts, readers and practitioners are referred to [4,12].

The most famous and frequently used EWMA chart under NP structure suggested by [13] for monitoring shifts in the process target/median was based on a simple sign test statistic. Due to the sensitive nature of the EWMA chart toward small and persistent shifts, different modifications have already been proposed and tested in the non-parametric exponentially weighted moving average 
(NPEWMA) charting structure. The authors of [14] designed an NPEWMA chart that was based on an SR test (NPEWMA-SR) for monitoring small and persistent shifts in the process target/median. A NP synthetic EWMA (SynEWMA) sign chart was proposed by [15] that improved the sensitivity of NPEWMA with the conforming RL chart. For more information on NP extended EWMA charts, readers and practitioners are referred to $[16,17]$ and the references therein. All the above-mentioned extensions were based on simple random sampling (SRS) scheme, whereas the charts based on the ranked set sampling (RSS) scheme are more sensitive in nature and are often more economical. A lot of work needs to be done in this area to offer more sensitive and economical design structures.

In the modern monitoring schemes, financial control has become a key point for quality practitioners that are used for reducing the quality cost. Quality engineers are always trying to minimize the difference between actual and budgeted cost. The SRS technique is frequently used in SPC but sometimes it has a high cost and provides less efficient results. In such situations, practitioners need a better alternative to SRS. The RSS technique provides efficient results and is also helpful for reducing the prevention cost.

The idea of RSS was initiated by [18] in situations when measurements are very expensive or of a distractive nature. In real life, it is apparent in many situations that the actual measurements of datasets are very expensive, while the visual inspection done by an expert (perfect ranking) is easy and inexpensive. As an example, in different manufacturing processes, the actual measurements of each quantity are costly and time consuming, while the visual inspection can save time and is relatively inexpensive. In control charting, RSS schemes are mostly used to increase the sensitivity of the monitoring process. RSS estimators are more efficient and unbiased as compared to the SRS scheme. The sample selection criteria under RSS schemes are not problematic. The criteria have some similarities to stratified random sampling, in which we divide the whole population into different subgroups where within groups objects are homogeneous, and between, they are heterogeneous. The following steps are involved to draw a random sample of size $n$ using the RSS scheme. (i) Draw an $n^{2}$ size of samples from the targeted population/process. (ii) Divide these samples into $n$ subgroups in an arbitrary way where each sample has size $n$. (iii) Rank each observation given in samples in ascending order. (iv) Choose the first observation from the first sample, the second observation of the second sample, and the last observation of the last sample. (v) It provides a ranked set sample of size $n$ with $m=1$ cycle.

The efficiency of the control chart is mostly enhanced by using Fast Initial Response (FIR) (cf. [19]) Auxiliary information (cf. [20,21]) and RSS scheme (cf. [22-25]).

This study intends to increase the sensitivity of the NPEWMA-SN chart based on the SN test statistic developed by [26] using an RSS scheme (NPREWMA-SN chart hereafter). The proposed NPREWMA-SN chart is developed under a process of standard normal, student's $t$, logistic, Laplace, and contaminated normal distributions. The main purpose of this study is to develop a simple, sensitive and inexpensive chart for monitoring the small deviations in the process location. In this article, the median is used as a target value because the mean has great deficiencies in the presence of outliers (cf. $[27,28])$. The key advantages of the proposed NPREWMA-SN chart over its counterparts can be described as follows. (i) There is no need of symmetry assumption for the proposed scheme (as is required for some others). (ii) The proposed scheme can be used to monitor the known mean, median, or percentiles of the distribution. (iii) The proposed scheme can also be performed efficiently for heavy tailed distributions. (iv) There is no need for actual measurements of observations, just a need for the information that the actual measurement is greater or less than the targeted value. (v) There is no need for process variance specification. (vi) It is easy to find the IC and OOC distribution of the sign (SN) statistic that is binomial. (vii) The efficiency of the designed NPREWMA-SN method is compared with its existing counterparts and it is found to be superior.

The remaining article is outlined as follows. Section 2 provides the structure formulation of the proposed chart. Section 3 describes the estimation procedures of the design parameters. Section 4 deals with RL evaluations and robustness. The OOC performance of the proposed chart is discussed 
in Section 5. Section 6 provides extensive comparisons of the NPREWMA-SN chart with its existing competitors. Section 7 illustrates a numerical example using a real dataset. The article ends with a summary and concluding remarks.

\section{Design Structure of NPEWMA-SN and the Proposed NPREWMA-SN Schemes}

This section describes the design structures of NPEWMA-SN and the proposed NPREWMA-SN charts based on an efficient sign test statistic described by [26].

\subsection{The Non-Parametric EWMA-SN Chart}

Suppose a quality characteristic $Y_{q j}$ has values $Y_{q 1}, Y_{q 2}, \ldots, Y_{q n}$, where $q=1,2,3, \ldots$ represent the coherent subgroups of independent observations of size $n>1$. The sampled process has unknown continuous distribution with median $\left(M_{0}\right)$ as a targeted value. The process is considered IC, when the process median is equal to the targeted value $M_{0}$ (cf. [29]). Take all possible differences $\left(Y_{q j}-M_{0}\right)$; then, the sign statistic $S N_{q}$ can take the values as:

$$
\operatorname{sign}\left(Y_{q j}-M_{0}\right)=\left\{\begin{array}{cl}
1 & \text { if, } Y_{q j}-M_{0}>0 \\
0 & \text { if, } Y_{q j}-M_{0}=0 \\
-1 & \text { if, } Y_{q j}-M_{0}<0
\end{array}\right\}
$$

To find the exact distribution of $S N_{q}$ we have $M_{q}=\frac{n+S N_{q}}{2}$, where $M_{q}$ follows binomial distribution with $E\left(M_{q}\right)=n p$ and $\operatorname{var}\left(M_{q}\right)=n p(1-p)$, where $p$ is the probability of success. $S N_{q}=n^{+}-n^{-}$; here, $n^{+}$shows the number of positive signs and $n^{-}$represents the number of negative signs. It is considered $P\left(Y_{q j}=M_{0}\right)=0$, as the process follows continuous distributions. Using this transformation, we have $S N_{q}=2 M_{q}-n$ with mean, $E\left(S N_{q}\right)=n(2 p-1)$, and variance, $\operatorname{Var}\left(S N_{q}\right)=4 n p(1-p)$, where $p$ is the probability of the process median that is equal to 0.5 (cf. $[4,26]$ ).

The NPEWMA-SN using the $S N_{q}$ statistic can be deifned as:

$$
E W M A_{q}=\lambda\left(S N_{q}\right)-(1-\lambda) E W M A_{q-1}
$$

where $\lambda(0<\lambda \leq 1)$ is the smoothing constant that is used to assign the weights to the current and previous observations. The mean and variance of $E W M A_{q}$ are, respectively, $n(2 p-1)$ and $\frac{\lambda}{2-\lambda} 4 n p(1-p)$. Using these quantities, the corresponding control limits of the NPEWMA-SN chart are:

$$
\left.\begin{array}{ccc}
U C L_{E W M A_{q}} & = & n(2 p-1)+L \sqrt{\frac{\lambda}{2-\lambda} 4 n p(1-p)}, \\
C L_{E W M A_{q}} & = & n(2 P-1), \\
L C L_{E W M A_{q}} & = & n(2 p-1)-L \sqrt{\frac{\lambda}{2-\lambda} 4 n p(1-p)}
\end{array}\right\} .
$$

If the $E W M A_{q}$ statistic defined in Equation (2) falls within the control limits expressed in Equation (3), the process is declared IC; otherwise, OOC.

\subsection{The Proposed Non-Parametric REWMA-SN Chart}

The proposed scheme is used to enhance the efficiency of the NPEWMA-SN chart by applying an RSS scheme. Suppose a quality characteristic $Y_{q, j(h)}$ is under study, with a target value $M_{0}$, where $q=1,2,3, \ldots$, each sample has $j=1,2,3, \ldots, n$ values and $h=1,2,3, \ldots, m$ number of cycles used in an RSS scheme. In an RSS scheme, the sample size $n$ with $m$ number of cycles can take the $r=m n$ iteration. The sample selection criteria under an RSS scheme can be elaborated as:

Cycle 1: $n \times n$ Ranked observations 


$$
\begin{array}{ccccc}
Y_{1,1(1)}, & Y_{1,2(1)}, & Y_{1,3(1)}, & \ldots, & Y_{1, n(1)} \\
Y_{2,1(1)}, & Y_{2,2(1)}, & Y_{2,3(1)} & \ldots, & Y_{2, n(1)} \\
Y_{3,1(1)}, & Y_{3,2(1)}, & Y_{3,3(1)}, & \ldots, & Y_{3, n(1)} \\
& \vdots & & \\
Y_{n, 1(1)}, & Y_{n, 2(1)}, & Y_{n, 3(1)}, & \ldots, & Y_{n, n(1)}
\end{array}
$$

Cycle 2: $n \times n$ Ranked observations

$$
\begin{array}{ccccc}
Y_{1,1(2)}, & Y_{1,2(2)}, & Y_{1,3(2)}, & \ldots, & Y_{1, n(2)} \\
Y_{2,1(2)}, & Y_{2,2(2)}, & Y_{2,3(2)} & \ldots, & Y_{2, n(2)} \\
Y_{3,1(2)}, & Y_{3,2(2)}, & Y_{3,3(2)}, & \ldots, & Y_{3, n(2)} \\
& \vdots & & \\
Y_{n, 1(2)}, & Y_{n, 2(2)}, & Y_{n, 3(2)}, & \ldots, & Y_{n, n(2)}
\end{array}
$$

Cycle m: $n \times n$ Ranked observations

$$
\begin{array}{ccccc}
Y_{1,1(m)}, & Y_{1,2(m)}, & Y_{1,3(m)}, & \ldots, & Y_{1, n(m)} \\
Y_{2,1(m)}, & Y_{2,2(m)}, & Y_{2,3(m)}, & \ldots, & Y_{2, n(m)} \\
Y_{3,1(m)}, & Y_{3,2(m)}, & Y_{3,3(m)}, & \ldots, & Y_{3, n(m)} \\
& \vdots & & \\
Y_{n, 1(m)}, & Y_{n, 2(m)}, & Y_{n, 3(m)}, & \ldots, & Y_{n, n(m)}
\end{array}
$$

The possible differences of $Y_{q j(h)}$ from their target $M_{0}$ can be defined as $Y_{q j(h)}-M_{0}$; that can takes the values as

$$
\operatorname{sign}\left(Y_{q j(h)}-M_{0}\right)=\left\{\begin{array}{cl}
1 & \text { if, } Y_{q j(h)}-M_{0}>0 \\
0 & \text { if }, Y_{q j(h)}-M_{0}=0 \\
-1 & \text { if }, Y_{q j(h)}-M_{0}<0
\end{array}\right\} .
$$

We know that $M_{q}=\frac{n+S N_{q}}{2}$; here, in the RSS scheme, the total number of iterations are denoted by $r$ instead of $n$ and $S N_{q}$ replaced with $R S N_{q}$ so, $R M_{q}=\frac{r+R S N_{q}}{2}$, where $R S N_{q}=r^{+}-r^{-}$. Here, $r^{+}$shows positive signs and $r^{-}$presents negative signs in a ranked set subgroup, respectively. It is assumed that $P\left(Y_{q j(h)}=M_{0}\right)=0$, as process follows continuous distributions. The statistic $R M_{q}$ follows binomial distribution with mean, $E\left(R M_{q}\right)=r p$, and variance, $\operatorname{Var}\left(R M_{q}\right)=r p(1-p) \varphi_{0}^{2}$. Using this information, we can find the mean and variance of $R S N_{q}$ that is, $E\left(R S N_{q}\right)=r(2 p-1)$ and $\operatorname{Var}\left(R S N_{q}\right)=4 r p(1-p) \varphi_{0}^{2}$, respectively, where the quantity $\varphi_{0}^{2}$ reveals the advantages of RSS over SRS and is taken from [30].

The NPREWMA-SN using the $R S N_{q}$ statistic can be defined as:

$$
R E W M A_{q}=\lambda\left(R S N_{q}\right)-(1-\lambda) R E W M A_{q-1}
$$

where $\lambda(0<\lambda \leq 1)$ is the smoothing constant that is used to assign the weights to the current and previous observations. To find the control limits, the mean and variance are the essential part of the limits. So, the mean of the NPREWMA-SN chart is $r(2 p-1)$ and its variance is $\frac{\lambda}{2-\lambda} 4 r p(1-p) \varphi_{0}^{2}$. Using these measurements, the corresponding control limits of the NPREWMA-SN chart are given as:

$$
\left.\begin{array}{ccc}
U C L_{\text {REWMA }_{q}}= & r(2 p-1)+L_{1} \sqrt{\frac{\lambda}{2-\lambda} 4 r p(1-p) \varphi_{0}^{2}} \\
C L_{\text {REWMA }}= & r(2 p-1) \\
L C L_{\text {REWMA }}= & r(2 p-1)-L_{1} \sqrt{\frac{\lambda}{2-\lambda} 4 r p(1-p) \varphi_{0}^{2}}
\end{array}\right\} .
$$


where ' $L_{1}$ ' is the control limits coefficient and the other terms are as defined earlier. The proposed scheme has three parameters: $U C L_{R E W M A_{q}}, C L_{R E W M A_{q}}$, and $L C L_{R E W M A_{q}}$ respectively. The chart triggers OOC situations if the NPREWMA-SN statistic given in Equation (5) falls outside the control limits (Equation (6)); otherwise, the process is assumed to be IC.

\section{Evaluation}

The measures used for evaluating and comparing the proposed scheme are the average RL (ARL), standard deviation RL (SDRL), $\mathrm{P}_{5}, \mathrm{P}_{25}$ (first quartile $\mathrm{Q}_{1}$ ), $\mathrm{P}_{50}$ (median RL(MRL)), $\mathrm{P}_{75}$ (3rd quartile $\mathrm{Q}_{3}$ ), and $P_{95}$. ARL is a statistical measure used as a yardstick to assess RL properties of the NPREWMA-SN method. The total number of sample observations before a chart alarms is defined as RL and the mean of the RL distribution is called ARL. The IC ARL of a chart is denoted by $\mathrm{ARL}_{0}$ and OOC is nominated as $A R L_{1}$. A chart is considered efficient that has a smaller $A_{R L}$ at a specific shift. The ARL plays a leading role in RL evaluation but, due to the skewed nature of RL distribution, important information may be missed if all the aforementioned measures have not been considered. The RL characteristics of the proposed chart are calculated using the Monte Carlo simulation method, and codes are developed in R programming language applying 50,000 iterations. The Monte Carlo simulation is used and the complete algorithm to find a nominal $\mathrm{ARL}_{0}$ is as follows:

(i) Using an RSS scheme, we generate subgroups of size $n$ and repeat them for $m$ cycles to acquire $r=m n$ from continuous distributions that are under study.

(ii) Choose the values of smoothing parameter $\lambda$ and $L_{1}$ for a specified $A R L_{0}$ and shift size.

(iii) Perform the NPREWMA-SN plotting statistic given in Equation (5) at each subgroup.

(iv) Construct the control limits given in Equation (6) and plot the NPREWMA-SN statistic used in step (iii) on these control limits.

(v) Observe the process until an OOC point appears.

(vi) Fixed the value of the multiplier $\left(L_{1}\right)$ at the required $\operatorname{IC~ARL}_{0}(370,500)$.

(vii) Repeat these steps 50,000 times and estimate the RL profiles of the process.

Selection of Design Parameters $m, n, \lambda$, and $L_{1}$ and Implementation of the Proposed Scheme

The proposed scheme has four design parameters $\left(m, n, \lambda\right.$, and $\left.L_{1}\right)$, where $m$ represents the number of cycles used in the RSS scheme, $n$ represents the size of the subgroups, $\lambda$ is the smoothing parameter, and $L_{1}$ is the size of the control limits (also known as a multiplier of the control limits). These parameters play a vital role in obtaining the optimal RL profiles of the proposed scheme. As mentioned above, a chart is considered more efficient that has a smaller value of $A R L_{1}$ at a specific shift using a fixed value of $\mathrm{ARL}_{0}$. According to this rule, two steps are involved in the selection of design parameters. In first phase, those combinations of the design parameters $m, n$ and $L_{1}$ are selected that give desired fixed values of $\mathrm{ARL}_{0}$ i.e., $(100,168,370$, or 500$)$. Then, the value of the smoothing parameter is finalized that gives a minimum $\mathrm{ARL}_{1}$ at a specific shift in the process location. It is understood that the EWMA chart performs effectively at small values of smoothing parameter $\lambda$, but large values can be used for the purpose of forecasting.

Using the above-mentioned criteria, all possible measures of the RL distributions are estimated for the proposed scheme with the combinations of design parameters $m \in\{1,2,3,4$ and 5$\}$, $n \in\{2,3,4,5,7$ and 10$\}, \lambda \in\{0.01,0.025,0.05,0.10,0.20\}$, and size of the limits $L_{1} \in\{2.0(0.1) 3.0\}$ that are reported in Table 1. 
Table 1. In-control (IC) run length (RL) profiles of the proposed chart when $m=3, n=3$.

\begin{tabular}{|c|c|c|c|c|c|}
\hline \multirow{2}{*}{ 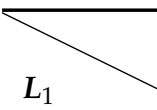 } & \multicolumn{2}{|c|}{ Small Shifts } & \multicolumn{2}{|c|}{ Moderate Shifts } & \multirow{2}{*}{$\begin{array}{c}\text { Large Shifts } \\
0.2\end{array}$} \\
\hline & 0.01 & 0.025 & 0.05 & 0.1 & \\
\hline \multirow{2}{*}{2} & $518.34(496.22)$ & $215.26(191.99)$ & $131.32(138.22)$ & $80.96(77.76)$ & $47.82(39.94)$ \\
\hline & $61,178,359,668,1513$ & $28,82,165,296,536$ & $14,42,88,164,400$ & $9,24,56,112,245$ & $6,17,36,68,134$ \\
\hline \multirow{2}{*}{2.1} & 614.27 (547.58) & $275.99(242.73)$ & $150.74(138.56)$ & $88.69(83.80)$ & $52.51(47.80)$ \\
\hline & $67,204,462,847,1629$ & $34,92,209,393,742$ & $16,54,106,212,403$ & $9,29,61,115,260$ & $6,18,39,70,153$ \\
\hline \multirow{2}{*}{2.2} & $795.38(758.44)$ & 353.936333 .8556 & $217.14(216.84)$ & $109.82(101.60)$ & $67.74(63.18)$ \\
\hline & $94273,547,1033,2344$ & $34,117,250,474,979$ & $20,64,136,282,650$ & $10,34,79,149,314$ & $6,22,48,94,191$ \\
\hline \multirow{2}{*}{2.3} & $949.18(1014.39)$ & $431.25(433.21)$ & $235.50(215.46)$ & $143.33(141.01)$ & $87.31(81.04)$ \\
\hline & $112,302,704,1280,2679$ & $4130,278,587,1281$ & $16,74,174,328,657$ & $13,49,100,180,401$ & $8,28,63,123,241$ \\
\hline \multirow{2}{*}{2.4} & $1236.62(1220.96)$ & $526.57(480.22)$ & $325.28(291.87)$ & $175.41(175.77)$ & 106.49 (113.55) \\
\hline & $119,354,819,1640,3989$ & $44,175,392,720,1455$ & $27,106,243,448,893$ & $15,53,120,230,534$ & $9,31,66,141,322$ \\
\hline \multirow{2}{*}{2.5} & $1512.44(1493.20)$ & $643.01(608.66)$ & $381.74(376.42)$ & $228.38(217.32)$ & $144.17(133.72)$ \\
\hline & $139,428,983,2044,4278$ & $65,214,486,872,1776$ & $37,131,269,505,1148$ & $17,72,167,313,615$ & $13,47,110,198,420$ \\
\hline \multirow{2}{*}{2.6} & $1729.36(1636.16)$ & 839.05 (747.32) & $502.70(492.03)$ & $297.66(294.60)$ & 197.65 (187.87) \\
\hline & $163,579,1216,24585170$ & $73,296,634,1151,2484$ & $39,150,346,661,1552$ & $23,100,212,406,923$ & $17,64,130,280,585$ \\
\hline \multirow{2}{*}{2.7} & $2537.12(2379.59)$ & $1117.22(1089.52)$ & $630.38(607.58)$ & $348.55(342.01)$ & $245.12(234.42)$ \\
\hline & $250,804,1836,3657,7277$ & $92,337,750,1487,3635$ & $42,181,455,883,1877$ & $23,106,251,481,1041$ & $15,74,177,353,722$ \\
\hline \multirow[b]{2}{*}{2.8} & $3026.70(2902.69)$ & $1337.90(1238.66)$ & $904.67(871.23)$ & $482.82(435.74)$ & $310.38(274.83)$ \\
\hline & $206,879,2125,4297,8879$ & $112,403,925,2020,3978$ & $60,282,646,1255,2586$ & $36,155,365,684,1322$ & $25,109,228,448,904$ \\
\hline \multirow{2}{*}{2.9} & 3791.593647 .701 & $1953.25(2198.28)$ & $1083.47(1021.30)$ & $598.71(521.07)$ & $418.96(428.51)$ \\
\hline & $2781009,2677,5397,10,838$ & $93,533,1229,2712,6189$ & $55,359,862,1590,2858$ & $47,191,476,807,1685$ & $17,106,283,591,1330$ \\
\hline \multirow[b]{2}{*}{3} & $4960.91(5137.9)$ & $2503.90(2747.66)$ & $1395.54(1371.04)$ & $809.23(720.13)$ & $575.25(567.95)$ \\
\hline & $308,1313,3598,6782,15,295$ & $123,584,1722,3503,7158$ & $82,449,961,1894,4183$ & $45,271,590,1122,2379$ & $39,178,372,795,1678$ \\
\hline
\end{tabular}


The first line of Table 1 shows the evaluated values of $\mathrm{ARL}_{0}$ and $\mathrm{SDRL}_{0}$, while $\mathrm{P}_{5}, \mathrm{P}_{25}, \mathrm{P}_{50}=\mathrm{MRL}$, $P_{75}$, and $P_{95}$ are reported in the 2 nd line of the table, respectively, where $P_{k}(k=1,2,3, \ldots, 100)$ are the percentile values of the RL distribution (cf. Table 1). It can be seen in Table 1 that at a fixed value of the smoothing parameter $(\lambda)$, the values of IC RL (reported in Table 1 ) of the proposed scheme are rapidly increasing as the value of $L_{1}$ increases. For example, the $A R L_{0}$ is 518.34 at $L_{1}=2$; when $L_{1}$ is increased from 2 to 3 , the $\mathrm{ARL}_{0}$ becomes 4960.91 for $\lambda=0.01$. This difference is very high at small values of $\lambda$ but relatively low at large values of $\lambda$. These results confirm the theory that the EWMA chart performs better at small values of $\lambda$. Table 1 is also helpful in identifying the shape of the RL distributions, where $\mathrm{ARL}_{0}>\mathrm{MRL}_{0}$, which indicates that the RL distributions are positive skewed. If a quality practitioner want to fix the desired $\mathrm{ARL}_{0}$, these values are very helpful to select the values of design parameters $\lambda$ and $L_{1}$. For example, to fix $A R L_{0} \cong 370$, when $\lambda=0.05$, the value of $L_{1}$ should lie between $2.4<L_{1}<2.5$ at $m=3$ and $n=3$ (cf. Table 1 ).

The values of $\mathrm{ARL}_{0}$ are plotted in Figure 1 at five small and moderate values of $\lambda$ that give an attractive visual look to easily understand the effect of $L_{1}$ on $\mathrm{ARL}_{0}$. It can be observed from Figure 1 that as we increase the value of $L_{1}$, the values of $\mathrm{ARL}_{0}$ increase and give the highest $\mathrm{ARL}_{0}$ at $\lambda=0.01$.

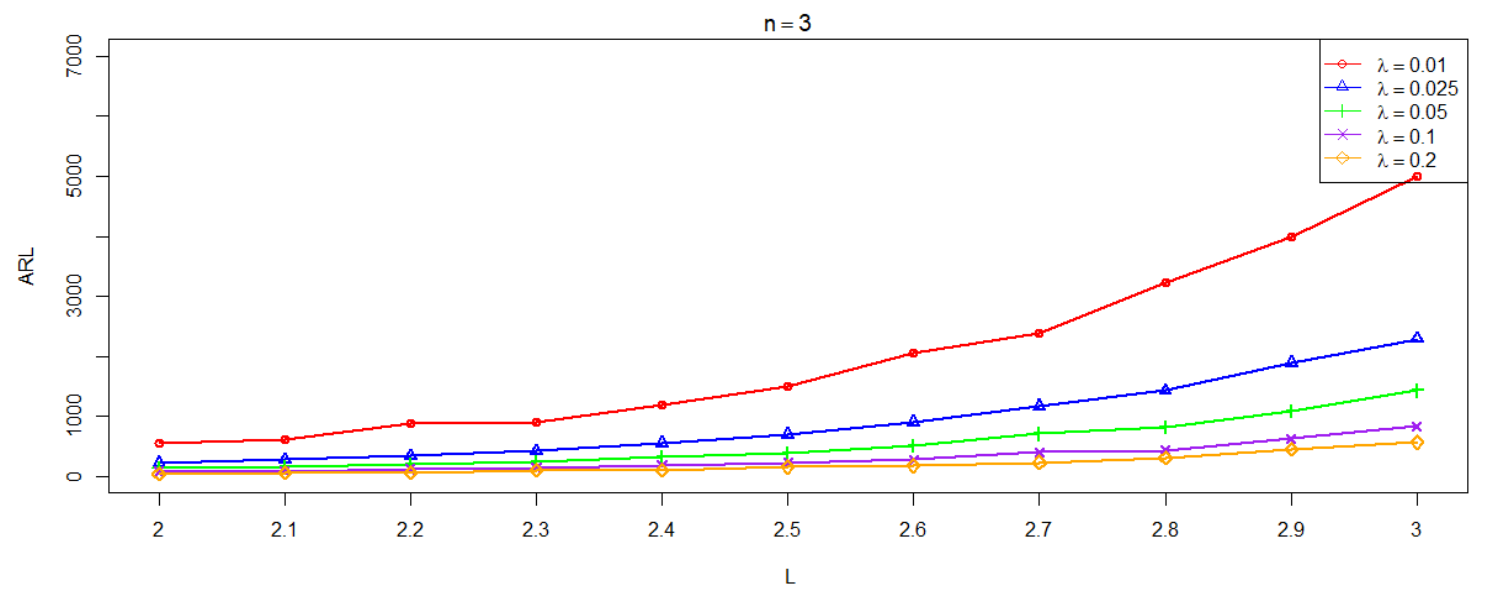

Figure 1. IC average RL (ARL) of the proposed chart for various $\left(\lambda\right.$ and $\left.L_{1}\right)$ under $m=3$ and $n=3$. Using the information given in Table 1, values of $L_{1}$ are reported in Table 2 with different combinations of $m, n$, and $\lambda$ at a nominal value of $\mathrm{ARL}_{0} \cong 370$ (cf. Table 2). For practical implementation of the proposed scheme, these values are very useful for the practitioners. For example, a quality practitioner want to spot the small and moderate shifts in the process location; using the proposed scheme, he must take $\left(\lambda, L_{1}\right)=(0.05,2.492)$ to fix $\mathrm{ARL}_{0} \cong 370$, at $m=3$ and $n=3$ (cf. Table 2).

Table 2. Values of $L_{1}$ having various combinations of $\lambda, m$, and $n$ with IC ARL $\cong 370$.

\begin{tabular}{ccccccc}
\hline \multirow{2}{*}{$\boldsymbol{m}$} & $\boldsymbol{n}$ & \multicolumn{5}{c}{$\boldsymbol{\lambda}$} \\
\cline { 3 - 7 } & & $\mathbf{0 . 0 1}$ & $\mathbf{0 . 0 2 5}$ & $\mathbf{0 . 0 5}$ & $\mathbf{0 . 1}$ & $\mathbf{0 . 2}$ \\
\hline & 2 & 1.831 & 2.219 & 2.492 & 2.684 & 2.822 \\
& 3 & 1.83 & 2.218 & 2.489 & 2.683 & 2.822 \\
1 & 4 & 1.829 & 2.217 & 2.487 & 2.682 & 2.821 \\
& 5 & 1.828 & 2.216 & 2.486 & 2.681 & 2.821 \\
& 7 & 1.827 & 2.215 & 2.485 & 2.68 & 2.82 \\
& 10 & 1.826 & 2.214 & 2.486 & 2.679 & 2.819 \\
\hline & 2 & 1.832 & 2.22 & 2.494 & 2.686 & 2.827 \\
& 3 & 1.831 & 2.219 & 2.493 & 2.685 & 2.826 \\
2 & 4 & 1.83 & 2.218 & 2.492 & 2.684 & 2.825 \\
& 5 & 1.829 & 2.217 & 2.491 & 2.683 & 2.824 \\
& 7 & 1.828 & 2.216 & 2.49 & 2.682 & 2.823 \\
& 10 & 1.827 & 2.215 & 2.489 & 2.681 & 2.822 \\
\hline
\end{tabular}


Table 2. Cont.

\begin{tabular}{ccccccc}
\hline \multirow{2}{*}{$\boldsymbol{m}$} & $\boldsymbol{n}$ & \multicolumn{5}{c}{$\boldsymbol{\lambda}$} \\
\cline { 3 - 7 } & & $\mathbf{0 . 0 1}$ & $\mathbf{0 . 0 2 5}$ & $\mathbf{0 . 0 5}$ & $\mathbf{0 . 1}$ & $\mathbf{0 . 2}$ \\
\hline \multirow{4}{*}{3} & 2 & 1.833 & 2.223 & 2.495 & 2.689 & 2.829 \\
& 3 & 1.832 & 2.222 & 2.492 & 2.688 & 2.828 \\
& 4 & 1.831 & 2.221 & 2.491 & 2.687 & 2.827 \\
& 5 & 1.83 & 2.22 & 2.49 & 2.686 & 2.826 \\
& 7 & 1.829 & 2.219 & 2.489 & 2.685 & 2.825 \\
& 10 & 1.828 & 2.218 & 2.488 & 2.684 & 2.824 \\
\hline \multirow{4}{*}{4} & 2 & 1.834 & 2.224 & 2.496 & 2.691 & 2.831 \\
& 3 & 1.833 & 2.223 & 2.495 & 2.69 & 2.83 \\
& 4 & 1.832 & 2.222 & 2.494 & 2.689 & 2.829 \\
& 5 & 1.831 & 2.221 & 2.493 & 2.688 & 2.828 \\
& 7 & 1.83 & 2.22 & 2.492 & 2.687 & 2.827 \\
& 10 & 1.829 & 2.219 & 2.491 & 2.686 & 2.826 \\
\hline \multirow{4}{*}{5} & 2 & 1.835 & 2.225 & 2.497 & 2.693 & 2.833 \\
& 3 & 1.834 & 2.224 & 2.494 & 2.692 & 2.832 \\
& 4 & 1.833 & 2.223 & 2.492 & 2.691 & 2.831 \\
& 5 & 1.832 & 2.222 & 2.491 & 2.69 & 2.83 \\
& 7 & 1.831 & 2.221 & 2.491 & 2.689 & 2.829 \\
& 10 & 1.83 & 2.22 & 2.489 & 2.688 & 2.828 \\
\hline
\end{tabular}

\section{RL Evaluation and IC Robustness of the Proposed Scheme}

The performance of the proposed scheme has been evaluated under both the normal environment and non-normal environment. The continuous symmetric type distribution involved in this proposed study includes the following: (a) normal distribution with zero mean and unit variance, $\mathrm{N}(0,1)$ that has bell-shaped, mesokurtic symmetrical distribution; (b) Student's $t$-distribution with $(v=4)$ degrees of freedom that has heavy tail, platykurtic-shaped symmetrical distribution; (c) Laplace distribution, $\operatorname{DE}(0,1 / \sqrt{2})$ (double exponential distribution); (d) the logistic distribution, $\operatorname{LOG}(0, \sqrt{3} / \pi)$; (e) and the contaminated normal distribution $(\mathrm{CN})$ to monitor the behavior of the proposed scheme in the presence of the outlier. The detailed formulation of the $\mathrm{CN}$ distribution can be seen in [17]. All the above-mentioned distributions are adjusted with zero mean and unit standard deviation for easy comparisons. These distributions have been used to observe the IC robustness of the proposed scheme. The specific use of these distributions is to observe the behavior of the proposed chart in heavy-tailed and sharper peak distributions when most of the values of the datasets fall away from their mean (i.e., Student's $t$ distribution and logistics distribution). Meanwhile, the $\mathrm{CN}$ distribution is specially used to monitor the behavior of the proposed scheme in the presence of outliers. The density functions of the given distributions are given in Table 3.

Table 3. Density functions of the continuous distributions that are under study.

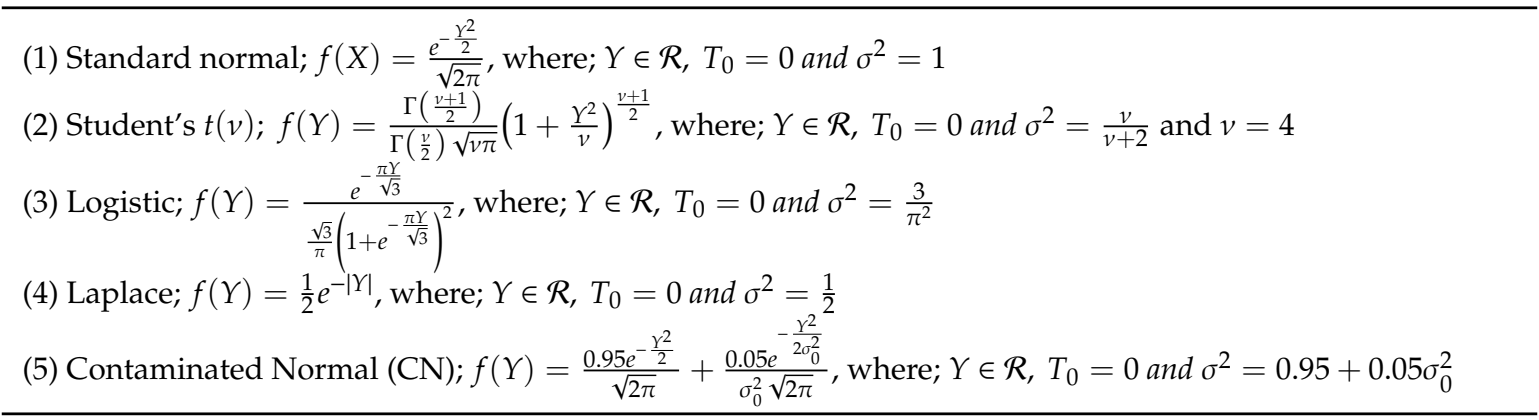




\section{IC Robustness of the Proposed Chart}

As has already been mentioned in Section 1, the non-parametric charts are IC robust for all the continuous distributions. Since the proposed scheme has also been designed under an NP structure, the IC RL distributions and other relevant properties of the proposed scheme should remain constant for all the given continuous distributions. The IC RL characteristics of the proposed and alternatives charts are estimated using the Monte Carlo simulation method with 50,000 iterations, and the results are tabulated in Table 4 . Five small and moderate values of $\lambda=0.01,0.025,0.05,0.10$ and 0.20 are used to check the IC robustness of the proposed scheme and the competitors. The values of $L_{1}$ are adjusted at $\mathrm{ARL}_{0} \cong 370$ for the proposed and NPEWMA-SN chart, while for the EWMA- $\bar{X}$ chart, these values are fixed at $A R L_{0} \cong 370$ under standard normal distribution $\mathrm{N}(0,1)$. $\mathrm{ARL}_{0}$ and $\mathrm{SDRL}_{0}$ are reported in the first line of Table 4 , while $\mathrm{P}_{5}, \mathrm{P}_{25}, \mathrm{P}_{50}=\mathrm{MRL}, \mathrm{P}_{75}$, and $\mathrm{P}_{95}$ are reported in the 2 nd line of Table 4, respectively (cf. Table 4).

Table 4. IC RL profiles of the proposed and its competitor chart when $m=1$ and $n=5$. CN: contaminated normal distribution, EWMA: exponentially weighted moving average, NPREWMA: non-parametric exponentially weighted moving average based on sign statistics under a ranked set sampling scheme.

\begin{tabular}{|c|c|c|c|c|c|}
\hline \multicolumn{6}{|c|}{ NPREWMA-SN } \\
\hline$\lambda, L_{1}$ & $(0.01,1.828)$ & $(0.025,2.215)$ & $(0.05,2.486)$ & $(0.1,2.681)$ & $(0.2,2.821)$ \\
\hline For all & $370.61(323.80)$ & $369.57(338.54)$ & $370.14(358.15)$ & $370.73(340.09)$ & $369.28(334.98)$ \\
\hline Distributions & $47,132,274,574,1015$ & $38,111,235,465,1031$ & $32,104,248,492,1084$ & $24,97,258,512,1030$ & $24,108,246,454,1001$ \\
\hline \multicolumn{6}{|c|}{ NPEWMA-SN } \\
\hline$\lambda, L$ & $(0.01,1.827)$ & $(0.025,2.229)$ & $(0.05,2.484)$ & $(0.1,2.682)$ & $(0.2,2.811)$ \\
\hline For all & 371.32 (334.36) & $369.08(347.85)$ & $372.68(360.56)$ & $368.18(365.56)$ & $371.45(366,43)$ \\
\hline Distributions & $50,132,270,505,1029$ & $38,121,363,505,1065$ & $32,116,263,510,1099$ & $24,106,255,500,1098$ & $24,113,261,525,1113$ \\
\hline \multicolumn{6}{|c|}{ EWMA $-\bar{X}$} \\
\hline Distributions & $(0.01,2.014)$ & $(0.025,2.314)$ & $(0.05,2.523)$ & $(0.1,2.719)$ & $(0.2,2.856)$ \\
\hline \multirow{2}{*}{$\mathrm{N}(0,1)$} & $370.41(476.77)$ & $370.04(407.82)$ & $370.50(386.42)$ & $371.01(366.88)$ & $369.66(365.41)$ \\
\hline & $2,25,189,551,1350$ & $4,76,248,539,1202$ & $8,96,253,527,1157$ & $14,105,261,523,1119$ & $18,106,252,504,1093$ \\
\hline \multirow{2}{*}{$t(4)$} & $382.20(486.02)$ & $374.44(414.72)$ & $358.89(369.93)$ & $321.37(325.56)$ & $248.98(249.82)$ \\
\hline & $2,30,199,555,1381$ & $3,71,242,535,1213$ & $6,92,246,504,1091$ & $11,90,220,449,970$ & $10,70,173,345,751$ \\
\hline \multirow{2}{*}{ Logistic } & $373.53(486.88)$ & $378.23(421.40)$ & $368.63(383.20)$ & $360.75(373.97)$ & $327.13(329.45)$ \\
\hline & $2,28,190,541,1339$ & $3,74,242,536,1218$ & $8,96,248,516,1136$ & $12,95,247,500,1090$ & $15,92,227,455,985$ \\
\hline \multirow{2}{*}{ Laplace } & $377.23(490.13)$ & $372.96(423.18)$ & $364.79(378.16)$ & $346.44(353.19)$ & $297.91(304.97)$ \\
\hline & $2,28,192,538,1374$ & $3,72,242,535,1186$ & $7,92,248,513,1133$ & $11,98,238,478,1058$ & $13,84,200,410,911$ \\
\hline \multirow{2}{*}{$\mathrm{CN}$} & $122.74(192.02)$ & $120.68(150.92)$ & $105.17(118.98)$ & $89.98(96.26)$ & $67.15(69.59)$ \\
\hline & $1,6,36,160,527$ & $1,12,64,174,428$ & $1,18,65,153,344$ & $2,21,60,126,284$ & $2,18,46,93,205$ \\
\hline
\end{tabular}

It can be observed that the IC RL properties of the proposed scheme are the same for all the values of $\lambda$, and they are also the same for all the continuous distributions under study including the $\mathrm{CN}$, which indicate the better resistance of the NP charts against the outlier. It can be evaluated from Table 4 that the NPREWMA-SN chart attains almost the exact standard value of $\mathrm{ARL}_{0} \cong 370$. Due to this quality of the proposed scheme, it is a better choice for practitioners because it attains almost exact nominal values of $\mathrm{ARL}_{0}$ (cf. Table 4 ).

The EWMA- $\bar{X}$ chart does not fulfill the IC robustness; also, it has larger variations in RL distributions. The IC RL properties vary dramatically under different continuous distributions. For example, keeping $\mathrm{ARL}_{0}$ as a yardstick measure of location, its value for $\mathrm{N}(0,1)$ is 371 , while for $t(4)$, it is 321 , and a dramatic change can be observed for CN distribution where its value is 89 , at $\lambda=0.10, m=1$ and $n=5$. In Table 4 , all the bold values of the EWMA- $\bar{X}$ chart confirm its deficiency in non-normal situations. Due to this reason, it may create problems for practitioners in terms of more false alarms.

The EWMA- $\bar{X}$ chart can be seen as inefficient and problematic for large values of $\lambda$, especially under $\mathrm{CN}$ distribution, which means it may become inaccurate in the presence of outliers. 


\section{OOC Performance of the NPREWMA-SN Chart}

To observe the efficacy of the NPREWMA-SN chart and its shift detection ability, OOC RL measurements are calculated under different situations. A chart is considered more efficient that has small OOC RL values at a specific shift. To monitor the OOC performance of the proposed chart, a number of small shifts $\{\delta=0.025,0.03,0.05$ and 0.075$\}$, moderate shifts $\{\delta=0.1,0.15,0.20,0.25$ and 0.75$\}$ and large shifts $\{\delta=1.0,1.5$ and 2.0$\}$ are introduced in the process target.

\subsection{Effect of Smoothing Parameter $(\lambda)$ on OOC ARL Performance}

Table 5 shows the results of $\mathrm{ARL}_{1}, \mathrm{SDRL}_{1}$, and MRL $\mathrm{M}_{1}$ performance for different values of $\lambda$ at fixed values of $m=3, n=3$, and $\mathrm{ARL}_{0} \cong 370$. These $\mathrm{OOC}$ values are calculated by introducing a number of small and moderate shifts in the process. The process is IC at target $\left(M_{0}\right)$, while OOC values are calculated by introducing the shifts in process target that is $M_{0}+\delta$. These results show the effect of $\lambda$ on OOC RL performances and are very helpful for choosing the best choice of $\lambda$. It can be observed from Table 5 that small values of $\lambda$ are suitable for small shifts and large values are a better choice for tracing large shifts. As we increase the value of $\lambda$, the EWMA- $\bar{X}$ chart performs similar to the Shewhart-type chart, and when $\lambda=1$, the EWMA-X $\bar{X}$ chart approaches the Shewhart chart exactly. For example, the proposed scheme detects a small shift $\delta=0.025$ after 235 observations and a large shift $\delta=2.0$ after 4 observations for $\lambda=0.01$, while these shifts are detected after 289 and 2 observations, respectively, for $\lambda=0.2$. These kinds of observations can also be seen at other values of $\lambda$ (cf. Table 5). This comparison is depicted in Figure 2 for attractive and easy understanding. In Figure 2, five different OOC ARL curves are drawn at $\lambda=0.01,0.025,0.05,0.1$ and 0.2, keeping other design parameters constant, to monitor the effect of $\lambda$. The effect of $\lambda$ is almost the same at moderate and large shifts, but a significant difference can be observed at small shifts. The sensitivity of the proposed chart toward small shifts increases as we use small values of $\lambda$.

Table 5. Out-of-control (OOC) RL profiles of the proposed chart under different values of $\lambda$ and $\mathrm{ARL}_{0} \cong 370$. ARL: average RL, MRL: median RL, SDRL: standard deviation RL.

\begin{tabular}{|c|c|c|c|c|c|c|c|c|c|c|c|c|c|c|c|}
\hline \multirow{2}{*}{$\lambda$} & \multirow{2}{*}{$L_{1}$} & \multirow{2}{*}{ Metrics } & \multicolumn{13}{|c|}{$\delta$} \\
\hline & & & 0.025 & 0.03 & 0.05 & 0.075 & 0.1 & 0.15 & 0.2 & 0.25 & 0.5 & 0.75 & 1 & 1.5 & 2 \\
\hline \multirow{3}{*}{0.01} & \multirow{3}{*}{1.841} & ARL & 235.05 & 206.92 & 121.75 & 76.41 & 54.60 & 34.43 & 25.47 & 20.17 & 10.12 & 7.03 & 5.69 & 4.46 & 4.04 \\
\hline & & SDRL & 201.34 & 170.11 & 91.20 & 45.78 & 28.51 & 14.31 & 9.15 & 6.54 & 2.17 & 1.26 & 0.76 & 0.54 & 0.20 \\
\hline & & MRL & 173 & 156 & 95 & 64 & 49 & 32 & 24 & 19 & 10 & 7 & 6 & 4 & 4 \\
\hline \multirow{3}{*}{0.025} & \multirow{3}{*}{2.252} & ARL & 253.65 & 222.14 & 132.30 & 79.76 & 53.79 & 31.52 & 22.26 & 17.17 & 8.27 & 5.80 & 4.63 & 3.60 & 3.12 \\
\hline & & SDRL & 226.61 & 200.76 & 103.90 & 57.19 & 34.73 & 16.60 & 9.35 & 6.38 & 2.09 & 1.11 & 0.77 & 0.53 & 0.32 \\
\hline & & MRL & 187 & 163 & 102 & 63 & 45 & 28 & 21 & 16 & 8 & 6 & 5 & 4 & 3 \\
\hline \multirow{3}{*}{0.05} & \multirow{3}{*}{2.492} & ARL & 273.21 & 232.16 & 144.78 & 84.88 & 57.99 & 31.07 & 20.56 & 15.34 & 6.95 & 4.83 & 3.84 & 3.09 & 3.00 \\
\hline & & SDRL & 263.00 & 219.32 & 129.23 & 69.42 & 43.29 & 18.38 & 10.24 & 6.69 & 1.96 & 1.06 & 0.71 & 0.29 & 0.04 \\
\hline & & MRL & 194 & 166 & 105 & 65 & 46 & 27 & 18 & 14 & 7 & 5 & 4 & 3 & 3 \\
\hline \multirow{3}{*}{0.1} & \multirow{3}{*}{2.682} & ARL & 283.38 & 254.02 & 163.03 & 102.96 & 65.66 & 32.86 & 20.82 & 14.57 & 5.94 & 3.92 & 3.10 & 2.37 & 2.06 \\
\hline & & SDRL & 268.60 & 247.02 & 157.48 & 92.22 & 56.18 & 25.23 & 13.46 & 7.74 & 2.05 & 1.02 & 0.69 & 0.49 & 0.23 \\
\hline & & MRL & 206 & 176 & 113 & 75 & 49 & 25 & 17 & 13 & 6 & 4 & 3 & 2 & 2 \\
\hline \multirow{3}{*}{0.2} & \multirow{3}{*}{2.838} & ARL & 289.02 & 290.02 & 210.01 & 131.23 & 92.26 & 42.77 & 24.49 & 15.81 & 5.48 & 3.43 & 2.65 & 2.09 & 2.00 \\
\hline & & SDRL & 283.06 & 285.29 & 199.86 & 119.65 & 85.45 & 35.32 & 18.75 & 10.87 & 2.38 & 1.04 & 0.66 & 0.29 & 0.06 \\
\hline & & MRL & 200 & 207 & 148 & 95 & 68 & 33 & 19 & 13 & 5 & 3 & 3 & 2 & 2 \\
\hline
\end{tabular}




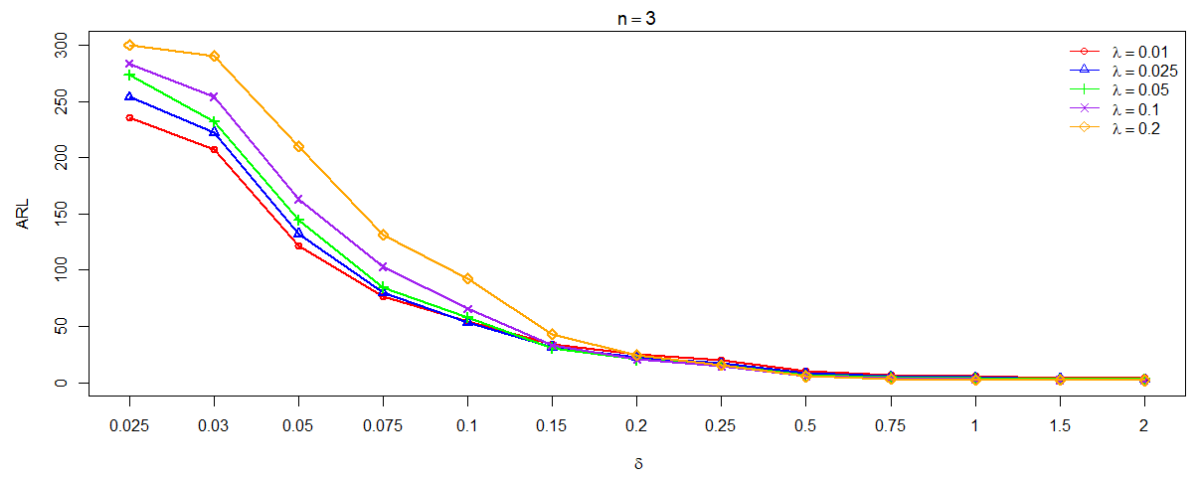

Figure 2. Effect of $\lambda$ on OOC ARL performance of the proposed chart under $m=3$ and $n=3$.

\subsection{Effect of Sample Size (n) on $A R L_{1}$ Performance}

The $\mathrm{ARL}_{1}$ values of the proposed scheme are calculated for different values of $n=2,3,4,5$ and 7 at $\lambda=0.05, m=1$, and these are depicted in Figure 3. These results show that the sensitivity of the proposed chart increases with the increase of sample size.

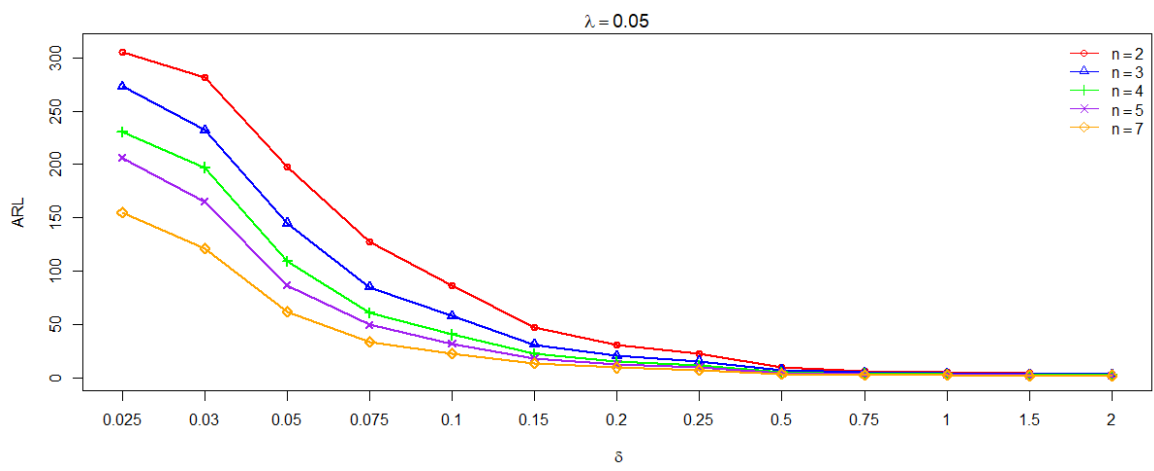

Figure 3. Effect of $n$ on OOC ARL performance of the proposed chart.

\subsection{Effect of Number of Cycles $(m)$ on OOC RL Performance of the Proposed Chart}

In an RSS scheme, it is better to increase number of cycles instead of the sample size to improve the efficiency of the chart. Five cycles $\{m=1,2,3,4$ and 5$\}$ are introduced in the proposed scheme, and their effect on $\mathrm{ARL}_{1}$ is presented in Figure 4. It can be observed in Figure 4 that an increase in $m$ improves the efficiency of the proposed scheme. The increase in sample size may be problematic for the practitioners as it involves high costs and extra time, but the RSS scheme provides an option to increase the number of cycles instead of the sample size. This effect can also be observed in Table 6 , where the effect of $m$ can be observed under different continuous distributions (cf. Table 6).

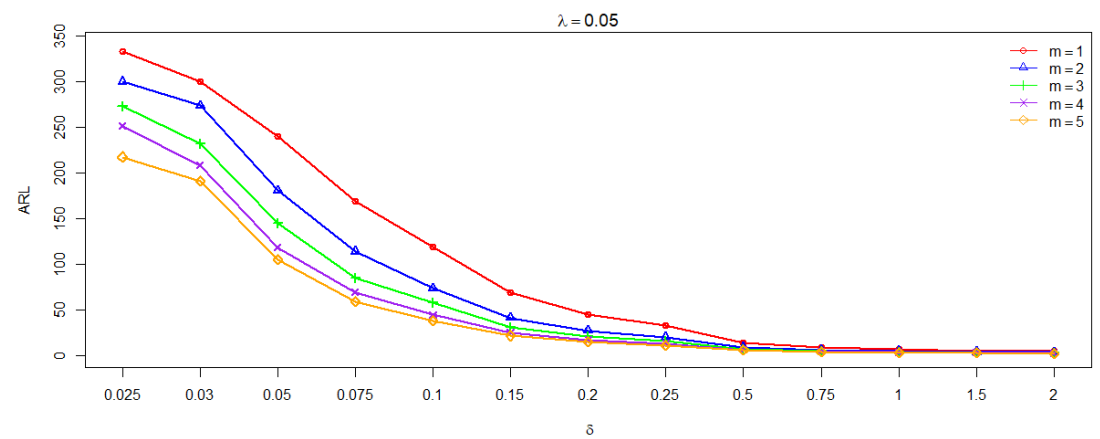

Figure 4. Effect of $m$ on $\mathrm{ARL}_{1}$ performance of the proposed chart under $\omega=0.05$. 
Table 6. OOC RL profiles of the proposed chart under different continuous distribution at $\lambda=0.05$ and $n=5$.

\begin{tabular}{|c|c|c|c|c|c|c|c|c|c|c|c|c|c|c|c|c|c|}
\hline \multirow{2}{*}{$m$} & \multirow{2}{*}{ Distributions } & \multirow{2}{*}{$L_{1}$} & \multirow{2}{*}{ Metrics } & \multicolumn{14}{|c|}{$\delta$} \\
\hline & & & & 0 & 0.025 & 0.03 & 0.05 & 0.075 & 0.1 & 0.15 & 0.2 & 0.25 & 0.5 & 0.75 & 1 & 1.5 & 2 \\
\hline \multirow{10}{*}{1} & \multirow{2}{*}{ Normal } & \multirow{10}{*}{2.484} & ARL & 371.14 & 267.67 & 265.03 & 166.21 & 109.49 & 70.43 & 38.79 & 25.59 & 19.16 & 8.34 & 5.68 & 4.49 & 3.72 & 3.33 \\
\hline & & & SDRL & 358.16 & 260.61 & 252.08 & 157.63 & 88.60 & 56.20 & 24.67 & 14.69 & 9.56 & 2.62 & 1.38 & 0.85 & 0.50 & 0.47 \\
\hline & \multirow{2}{*}{$\mathrm{t}(4)$} & & ARL & 369.04 & 296.22 & 262.21 & 187.58 & 114.35 & 76.43 & 42.03 & 29.03 & 20.94 & 9.31 & 6.23 & 4.90 & 3.98 & 3.64 \\
\hline & & & SDRL & 339.89 & 284.57 & 249.34 & 177.92 & 100.90 & 58.84 & 29.17 & 16.72 & 11.21 & 3.12 & 1.66 & 1.08 & 0.53 & 0.52 \\
\hline & \multirow{2}{*}{ Logistic } & & ARL & 371.36 & 269.84 & 243.68 & 154.52 & 89.71 & 58.12 & 31.98 & 22.08 & 16.74 & 7.46 & 5.22 & 4.30 & 3.68 & 3.34 \\
\hline & & & SDRL & 331.04 & 248.35 & 235.00 & 140.05 & 80.04 & 43.62 & 18.95 & 12.31 & 7.93 & 2.15 & 1.21 & 0.74 & 0.50 & 0.47 \\
\hline & \multirow{2}{*}{ Laplace } & & ARL & 369.27 & 200.76 & 161.50 & 87.32 & 49.13 & 33.38 & 20.08 & 14.67 & 11.37 & 6.17 & 4.64 & 4.01 & 3.52 & 3.26 \\
\hline & & & SDRL & 351.10 & 182.36 & 149.05 & 69.23 & 34.27 & 20.51 & 10.19 & 6.39 & 4.45 & 1.61 & 1.02 & 0.73 & 0.51 & 0.44 \\
\hline & \multirow{2}{*}{$\mathrm{CN}$} & & ARL & 369.25 & 291.35 & 261.09 & 181.05 & 109.42 & 74.56 & 40.28 & 27.15 & 20.01 & 8.62 & 5.86 & 4.64 & 3.75 & 3.39 \\
\hline & & & SDRL & 343.20 & 274.48 & 244.24 & 170.05 & 96.14 & 62.56 & 27.80 & 16.51 & 9.82 & 2.88 & 1.48 & 0.94 & 0.50 & 0.49 \\
\hline \multirow{10}{*}{2} & \multirow{2}{*}{ Normal } & \multirow{10}{*}{2.491} & ARL & 369.71 & 235.25 & 208.45 & 114.86 & 67.27 & 42.71 & 23.92 & 16.08 & 12.31 & 5.77 & 4.03 & 3.30 & 2.79 & 2.39 \\
\hline & & & SDRL & 351.87 & 227.80 & 195.13 & 95.15 & 52.67 & 30.17 & 13.55 & 7.50 & 4.78 & 1.49 & 0.79 & 0.50 & 0.43 & 0.49 \\
\hline & \multirow{2}{*}{$\mathrm{t}(4)$} & & ARL & 370.02 & 247.64 & 222.79 & 121.29 & 72.46 & 48.04 & 25.70 & 17.73 & 13.51 & 6.13 & 4.31 & 3.57 & 2.95 & 2.72 \\
\hline & & & SDRL & 355.90 & 238.03 & 202.73 & 109.21 & 53.08 & 33.75 & 13.82 & 8.25 & 5.55 & 1.63 & 0.86 & 0.61 & 0.38 & 0.45 \\
\hline & \multirow{2}{*}{ Logistic } & & ARL & 369.36 & 211.13 & 177.10 & 90.67 & 53.28 & 35.27 & 19.75 & 13.85 & 10.60 & 5.23 & 3.78 & 3.17 & 2.74 & 2.43 \\
\hline & & & SDRL & 337.04 & 193.66 & 160.40 & 75.77 & 39.36 & 22.59 & 9.84 & 5.68 & 3.89 & 1.27 & 0.68 & 0.45 & 0.44 & 0.50 \\
\hline & \multirow{2}{*}{ Laplace } & & ARL & 372.09 & 139.05 & 109.69 & 52.15 & 30.59 & 20.65 & 12.68 & 9.43 & 7.57 & 4.34 & 3.43 & 3.06 & 2.74 & 2.50 \\
\hline & & & SDRL & 355.74 & 121.29 & 91.11 & 39.01 & 18.27 & 10.91 & 5.44 & 3.25 & 2.47 & 0.89 & 0.57 & 0.40 & 0.44 & 0.50 \\
\hline & \multirow{2}{*}{$\mathrm{CN}$} & & ARL & 369.30 & 240.79 & 213.68 & 120.77 & 69.99 & 43.59 & 24.40 & 16.57 & 12.71 & 5.89 & 4.12 & 3.37 & 2.87 & 2.48 \\
\hline & & & SDRL & 353.03 & 217.37 & 202.58 & 105.09 & 55.13 & 30.42 & 13.38 & 7.61 & 5.39 & 1.52 & 0.80 & 0.55 & 0.37 & 0.50 \\
\hline \multirow{10}{*}{3} & \multirow{2}{*}{ Normal } & \multirow{10}{*}{2.49} & ARL & 372.13 & 202.70 & 169.80 & 85.81 & 50.60 & 31.53 & 18.02 & 12.67 & 9.69 & 4.67 & 3.28 & 2.79 & 2.12 & 2.00 \\
\hline & & & SDRL & 364.93 & 193.08 & 162.58 & 74.21 & 36.25 & 19.18 & 8.72 & 5.19 & 3.45 & 1.12 & 0.54 & 0.43 & 0.32 & 0.04 \\
\hline & \multirow{2}{*}{$\mathrm{t}(4)$} & & ARL & 372.22 & 220.77 & 179.18 & 97.89 & 54.04 & 34.77 & 19.49 & 13.55 & 10.35 & 5.06 & 3.50 & 3.00 & 2.37 & 2.08 \\
\hline & & & SDRL & 345.77 & 208.22 & 161.41 & 77.82 & 40.07 & 21.06 & 9.94 & 5.59 & 3.84 & 1.31 & 0.67 & 0.40 & 0.48 & 0.27 \\
\hline & \multirow{2}{*}{ Logistic } & & ARL & 369.50 & 174.36 & 144.93 & 72.39 & 40.31 & 26.08 & 15.50 & 10.88 & 8.35 & 4.13 & 3.09 & 2.70 & 2.07 & 2.00 \\
\hline & & & SDRL & 342.57 & 152.59 & 124.86 & 59.30 & 26.60 & 14.85 & 7.15 & 4.23 & 2.72 & 0.91 & 0.45 & 0.47 & 0.25 & 0.03 \\
\hline & I anlace & & ARL & 371.51 & 105.18 & 84.37 & 39.86 & 23.03 & 16.13 & 10.09 & 7.45 & 6.11 & 3.52 & 2.89 & 2.55 & 2.08 & 2.01 \\
\hline & Laplace & & SDRL & 372.55 & 87.47 & 66.81 & 26.64 & 12.49 & 7.35 & 3.53 & 2.32 & 1.67 & 0.66 & 0.41 & 0.50 & 0.28 & 0.09 \\
\hline & & & ARL & 369.25 & 206.74 & 172.76 & 90.08 & 51.19 & 33.47 & 18.80 & 13.13 & 10.01 & 4.76 & 3.35 & 2.85 & 2.16 & 2.01 \\
\hline & $\mathrm{CN}$ & & SDRL & 383.20 & 188.20 & 161.01 & 74.03 & 38.37 & 20.64 & 9.39 & 5.37 & 3.67 & 1.09 & 0.59 & 0.42 & 0.37 & 0.08 \\
\hline
\end{tabular}




\subsection{Distributional Effect on OOC RL Performance of the Proposed Chart}

The OOC ARL and SDRL values are calculated, and presented in Table 6, for all continuous distributions that are under study to measure the distributional effect on OOC RL performance. For this purpose, we used standard value of $\lambda=0.05$, with a sample size $n=5$ and different values of number of cycles $m=1,2$, and 3. It can be observed from Table 6 that the proposed scheme has almost the same IC performance in terms of ARL and SDRL but has little fluctuations in OOC performance. The results given in Table 6 also confirm the IC robust property of the proposed scheme. The IC ARL $\cong 370$ for each continuous distribution using the fixed values of design parameters. The results confirm that the estimated design parameters can be used for normal and non-normal situations. The fluctuation in OOC RL values is not too high, and minor changes can be observed in OOC performance of the proposed scheme. Under these circumstances, the proposed scheme is more sensitive toward small shifts in process location if the data comes from Laplace distribution (cf. Table 6).

\section{Comparisons}

To verify the superiority of the proposed scheme, it has been compared with alternatives NPEWMA-SN and EWMA- $\bar{X}$, under all continuous distributions that are under study for $\lambda=0.05$ and $n=5$. For comparison, ARL and SDRL are considered as indicator measures that are provided in Table 7. The percentage decrease (PD) in ARL is also a good indicator for easy understanding and is calculated as $\left(\frac{A R L_{0}-A R L_{1}}{A R L_{0}}\right) \times 100 \%$. Large values of PD in ARL indicate the outer performance of a control chart (cf. [17]).

\subsection{Proposed Versus NPEWMA-SN}

The ARL and SDRL values of the proposed and NPEWMA-SN chart proposed by [26] are given in Table 7 under different continuous distributions. For valid comparison, the RL values of both the charts are calculated using same design parameters that are $n=5, \lambda=0.05$ and IC $\mathrm{ARL}_{0} \cong 370$. If a $2.5 \%$ increase occurs in the process median, the proposed scheme has a $36.48 \%$ decrease in ARL in a normal situation, while the competitor NPEWMA-SN chart has only a 9.72\% decrease in ARL in a normal situation. The proposed scheme performs much better in situations where there are outliers in the data, and it can be observed in the performance of the proposed scheme under $\mathrm{CN}$ distribution. Under $\mathrm{CN}$ distribution, the proposed scheme detected the first OOC signal after 235.4 points at $\delta=0.025$, while the NPEWMA-SN chart detected this shift after 326.3 points. In Table 7, bold values of the proposed scheme show that it has better performance in a small and moderate shift detection than its competitor. The results of Table 7 demonstrate that at each value of $\delta$, the PD in ARL of the proposed scheme is better than its competitor. It is evident from Table 7 that the $\mathrm{ARL}_{1}$ and $\mathrm{SDRL}_{1}$ performances of the proposed chart are smaller than those of its counterpart under each continuous distribution that is under study. So, the proposed scheme is far better than the NPEWMA-SN scheme in the entire situation (cf. Table 7).

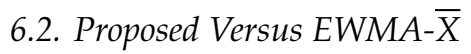

The calculated values of ARL and SDRL of the proposed and well-known classical EWMA- $\bar{X}$ charts under different continuous distributions are given in Table 7. Using design parameters $n=5$ and $\lambda=0.05$, the proposed scheme triggers first an OOC signal after 235.3 points, while its competitor triggers it after 298.3 points at $\delta=0.025$, under a normal environment. Using the same design parameters, the PD in ARL of the proposed chart is $31.62 \%$, while the value of EWMA- $\bar{X}$ is $18.38 \%$ at $\delta=0.025$ under heavy tailed student $t$-distribution. The above dominance of the proposed chart can also be observed on other small and moderate values of $\delta$. The large value of PD in ARL and small value of $A R L_{1}$ and $S D R L_{1}$ of the proposed chart is emphasizing that the proposed chart performs adequately to detect the nonconformities at small to moderate shifts in process target(s) (cf. Table 7). 
Table 7. Comparisons of the proposed and alternatives at $\lambda=0.05$ and $n=5$.

\begin{tabular}{|c|c|c|c|c|c|c|c|c|c|c|c|c|c|c|c|c|c|}
\hline \multirow{2}{*}{ Distributions } & \multirow{2}{*}{$L_{1}$} & \multirow{2}{*}{ Charts } & \multirow{2}{*}{ Metrics } & \multicolumn{14}{|c|}{$\delta$} \\
\hline & & & & 0 & 0.025 & 0.03 & 0.05 & 0.075 & 0.1 & 0.15 & 0.2 & 0.25 & 0.5 & 0.75 & 1 & 1.5 & 2 \\
\hline \multirow{6}{*}{$\mathrm{N}(0,1)$} & \multirow{2}{*}{2.491} & \multirow{2}{*}{ Proposed } & ARL & 369.71 & 235.25 & 208.45 & 114.86 & 67.27 & 42.71 & 23.92 & 16.08 & 12.31 & 5.77 & 4.03 & 3.30 & 2.79 & 2.39 \\
\hline & & & SDRL & 351.87 & 227.80 & 195.13 & 95.15 & 52.67 & 30.17 & 13.55 & 7.50 & 4.78 & 1.49 & 0.79 & 0.50 & 0.43 & 0.49 \\
\hline & \multirow{2}{*}{2.484} & \multirow{2}{*}{ NPEWMA-SN } & ARL & 369.45 & 334.85 & 306.71 & 240.01 & 167.55 & 119.16 & 65.92 & 43.77 & 31.76 & 12.91 & 8.42 & 6.56 & 5.01 & 4.38 \\
\hline & & & SDRL & 254.45 & 323.54 & 293.89 & 217.59 & 154.03 & 104.43 & 51.90 & 29.76 & 19.32 & 4.99 & 2.39 & 1.40 & 0.77 & 0.53 \\
\hline & \multirow{2}{*}{2.523} & \multirow{2}{*}{ EWMA-X } & ARL & 370.76 & 298.34 & 274.84 & 190.06 & 120.54 & 78.23 & 41.78 & 25.62 & 17.77 & 5.69 & 2.95 & 1.97 & 1.22 & 1.02 \\
\hline & & & SDRL & 386.42 & 307.34 & 284.28 & 193.44 & 118.22 & 72.20 & 35.36 & 20.36 & 13.29 & 3.69 & 1.68 & 1.01 & 0.44 & 0.15 \\
\hline \multirow{6}{*}{$\mathrm{t}(4)$} & \multirow{2}{*}{2.491} & \multirow{2}{*}{ Proposed } & ARL & 369.04 & 253.20 & 203.53 & 121.15 & 73.78 & 47.24 & 26.17 & 17.72 & 13.42 & 6.17 & 4.37 & 3.59 & 2.98 & 2.75 \\
\hline & & & SDRL & 339.89 & 230.25 & 191.89 & 101.25 & 58.91 & 33.16 & 14.80 & 8.20 & 5.66 & 1.65 & 0.93 & 0.65 & 0.35 & 0.44 \\
\hline & \multirow{2}{*}{2.484} & \multirow{2}{*}{ NPEWMA-SN } & ARL & 370.55 & 331.03 & 316.73 & 248.30 & 178.54 & 129.60 & 73.33 & 47.80 & 34.57 & 14.05 & 9.18 & 7.20 & 5.57 & 4.90 \\
\hline & & & SDRL & 368.44 & 315.17 & 297.07 & 229.46 & 160.66 & 111.41 & 58.77 & 33.63 & 21.50 & 5.74 & 2.81 & 1.72 & 0.97 & 0.72 \\
\hline & \multirow{2}{*}{2.535} & \multirow{2}{*}{ EWMA- $\bar{X}$} & ARL & 371.62 & 298.04 & 280.70 & 195.84 & 123.87 & 82.07 & 43.52 & 26.36 & 18.21 & 5.74 & 2.98 & 1.94 & 1.19 & 1.03 \\
\hline & & & SDRL & 387.67 & 309.16 & 288.43 & 193.18 & 118.50 & 75.87 & 36.49 & 20.97 & 13.95 & 3.64 & 1.67 & 0.97 & 0.42 & 0.16 \\
\hline \multirow{6}{*}{ Logistic } & \multirow{2}{*}{2.491} & \multirow{2}{*}{ Proposed } & ARL & 368.96 & 215.13 & 179.48 & 98.21 & 54.53 & 35.66 & 20.37 & 14.03 & 10.77 & 5.24 & 3.76 & 3.19 & 2.77 & 2.41 \\
\hline & & & SDRL & 342.82 & 196.74 & 162.90 & 87.11 & 40.25 & 23.67 & 10.62 & 5.92 & 3.98 & 1.25 & 0.67 & 0.44 & 0.43 & 0.49 \\
\hline & \multirow{2}{*}{2.484} & \multirow{2}{*}{ NPEWMA-SN } & ARL & 372.01 & 317.22 & 292.33 & 218.75 & 145.00 & 99.06 & 54.92 & 36.31 & 26.53 & 11.28 & 7.68 & 6.19 & 4.95 & 4.43 \\
\hline & & & SDRL & 358.51 & 302.16 & 273.39 & 204.65 & 124.91 & 83.71 & 40.07 & 23.29 & 15.06 & 3.99 & 1.98 & 1.24 & 0.74 & 0.55 \\
\hline & & & ARL & 368.02 & 305.19 & 274.26 & 190.67 & 122.02 & 80.02 & 41.87 & 25.52 & 17.95 & 5.66 & 2.96 & 1.99 & 1.21 & 1.03 \\
\hline & 2.526 & EWMA-X & SDRL & 377.56 & 320.91 & 286.08 & 196.29 & 117.82 & 73.75 & 35.14 & 20.13 & 13.43 & 3.64 & 1.68 & 1.04 & 0.45 & 0.17 \\
\hline & 401 & Pronosed & ARL & 371.09 & 139.05 & 109.69 & 52.15 & 30.59 & 20.65 & 12.68 & 9.43 & 7.57 & 4.34 & 3.43 & 3.06 & 2.74 & 2.50 \\
\hline & 2.491 & Iroposea & SDRL & 355.74 & 121.29 & 91.11 & 39.01 & 18.27 & 10.91 & 5.44 & 3.25 & 2.47 & 0.89 & 0.57 & 0.40 & 0.44 & 0.50 \\
\hline Laplace & 2484 & & ARL & 369.09 & 263.52 & 232.82 & 144.22 & 84.33 & 57.17 & 33.53 & 23.23 & 18.04 & 9.23 & 6.85 & 5.84 & 4.93 & 4.48 \\
\hline Laplace & 2.484 & NPEWMA-SN & SDRL & 350.38 & 251.89 & 217.23 & 128.16 & 68.76 & 42.64 & 20.98 & 12.44 & 8.47 & 2.84 & 1.58 & 1.08 & 0.75 & 0.57 \\
\hline & & & ARL & 373.22 & 298.10 & 283.04 & 190.91 & 121.81 & 82.38 & 42.22 & 26.14 & 17.99 & 5.74 & 2.96 & 1.97 & 1.21 & 1.03 \\
\hline & 2.535 & 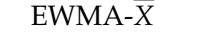 & SDRL & 397.05 & 308.46 & 289.74 & 193.51 & 119.42 & 76.01 & 35.67 & 20.56 & 13.40 & 3.60 & 1.68 & 1.00 & 0.45 & 0.17 \\
\hline & & Pronoced & ARL & 370.45 & 235.38 & 208.08 & 112.40 & 65.94 & 45.33 & 24.23 & 16.57 & 12.66 & 5.89 & 4.11 & 3.41 & 2.84 & 2.44 \\
\hline & 2.491 & Proposed & SDRL & 351.76 & 213.28 & 196.50 & 100.02 & 49.17 & 31.46 & 13.37 & 7.70 & 4.95 & 1.52 & 0.80 & 0.56 & 0.39 & 0.50 \\
\hline$C \mathrm{~N}$ & & & ARL & 368.36 & 326.26 & 306.96 & 255.16 & 173.34 & 117.28 & 72.01 & 45.44 & 32.24 & 13.40 & 8.63 & 6.70 & 5.16 & 4.51 \\
\hline $\mathrm{CN}$ & 2.484 & NPEWMA-SN & SDRL & 352.81 & 320.24 & 304.08 & 229.00 & 154.40 & 99.21 & 55.29 & 30.92 & 19.44 & 4.97 & 2.40 & 1.48 & 0.82 & 0.58 \\
\hline & 3198 & FWMA & ARL & 370.56 & 319.54 & 306.80 & 236.38 & 165.49 & 118.09 & 63.61 & 39.59 & 27.75 & 8.48 & 4.29 & 2.75 & 1.54 & 1.13 \\
\hline & 3.198 & EWMA & SDRL & 391.29 & 341.41 & 321.05 & 243.82 & 168.55 & 115.04 & 58.17 & 32.82 & 21.42 & 5.55 & 2.53 & 1.46 & 0.70 & 0.36 \\
\hline
\end{tabular}


For easy understanding, these comparisons are also presented in Figure 5. In this figure, the curves shows the OOC performance of the proposed and alternative charts against the different shifts. The figure captures the comparison of the proposed and competitors under four distributions. These OOC curves show that at large shifts, the performance of all the charts is almost the same but at small shifts, the proposed chart has the ability to detect quickly. As mentioned earlier, the proposed scheme has been designed for small and persistent shifts, and all the results given in the tables confirm the statement that the proposed scheme has a better ability to detect small shifts than its competitors.
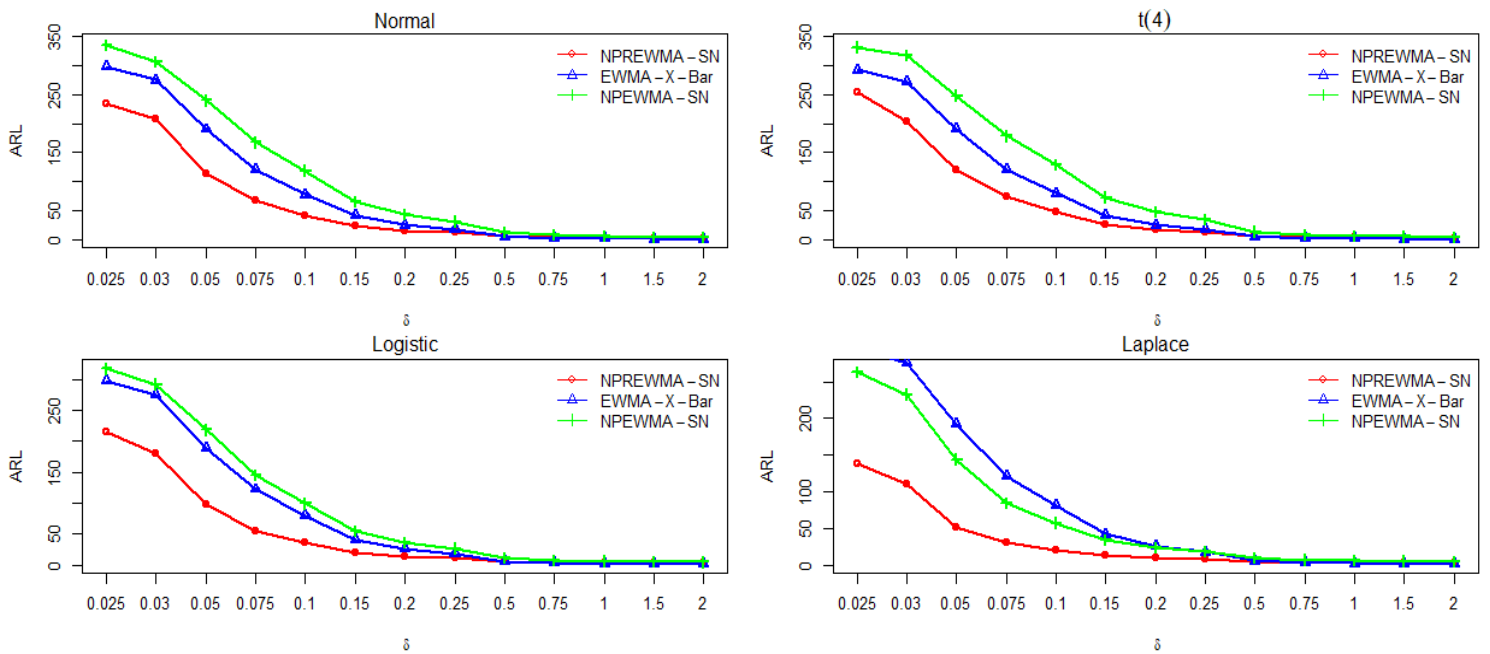

Figure 5. Overall comparisons of the proposed chart and its counterparts using $\mathrm{ARL}_{1}$ curves.

\section{Real Data Application of the Proposed Scheme}

For the illustration of the NPREWMA-SN chart, a real-life application is presented to observe the shift detection ability in the process monitoring. When manufacturing semiconductors, an important hard bake process is used with the combination of photolithography. The flow width of the resist in manufacturing substrate process is taken as a quality characteristic of interest. The different steps involved in this manufacturing process are displayed in Figure 6. To monitor this process, a sample of five wafers was taken every hour, and the average of flow width was computed. The dataset provided by [1] consists of the first 25 ICs and next 20 OOC sample observations of size 5 . We mixed 125 IC and 100 OOC observations to get 25 ranked set samples from IC and 20 from OOC observations of size $n=5$ with $m=3$ (cycles). From the resulting 45 samples, the first 25 samples are IC with a target value $M_{0}=1.5056$, while the remaining 20 samples are from the OOC process. From the Phase-I samples, the control limits of the proposed chart and its alternatives are computed. In the monitoring phase, the proposed and the competitors are also constructed.

For the implementation of the proposed and competitors, the design parameters for various charts are given as $n=15, L=2.485$ for NPEWMA-SN, $n=15, L=2.521$ for classical EWMA- $\bar{X}$, and $m=3, n=5, L_{1}=2.49$ for the proposed charts at fixed value of $\lambda=0.05$ and $\mathrm{IC}$ ARL $\cong 370$.

All the aforementioned charts are displayed in Figures 7-9. The proposed chart triggers the first OOC signal at the 38th measurement, while the NPEWMA-SN and classical EWMA- $\bar{X}$ control charts detect the first OOC signal at the 45th and 41st sample numbers, respectively (cf. Figures 7-9). The proposed scheme's quicker detection of an OOC signal provides valuable confirmation of its superiority over the other existing alternatives. 


\section{Silicon Wafers Manufacturing Process}

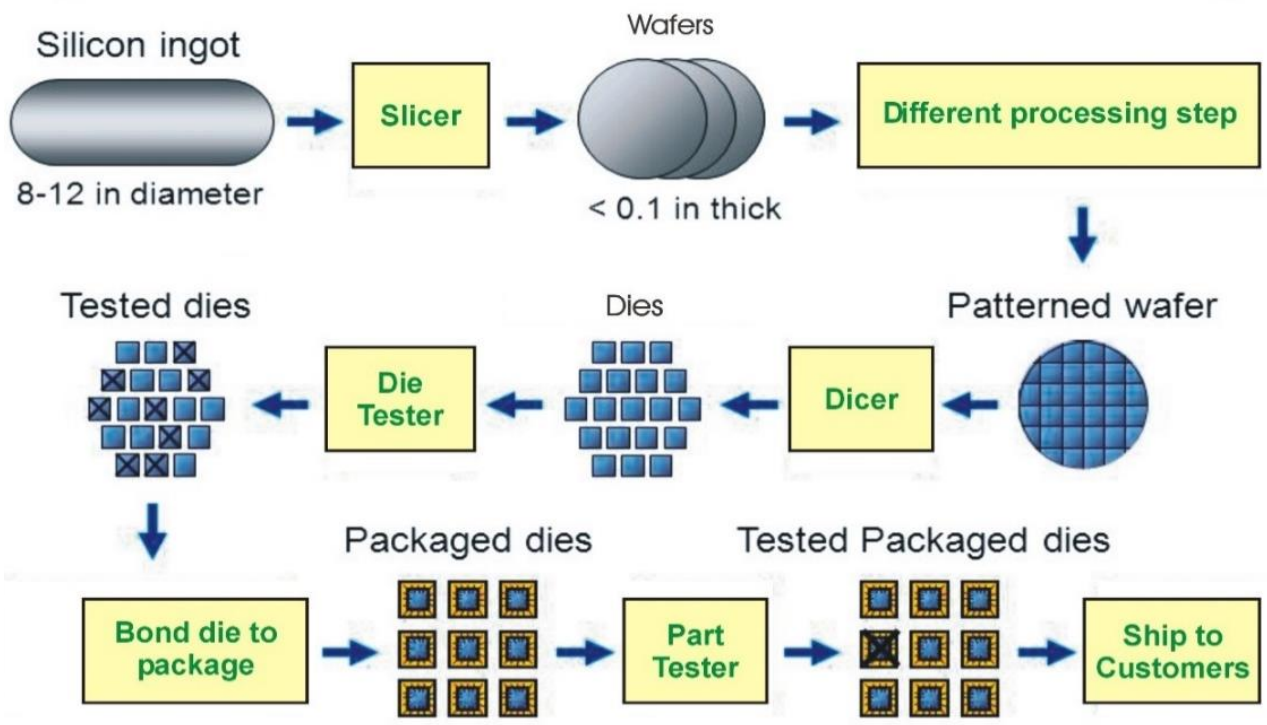

Figure 6. Manufacturing process of the basic silicon wafers.

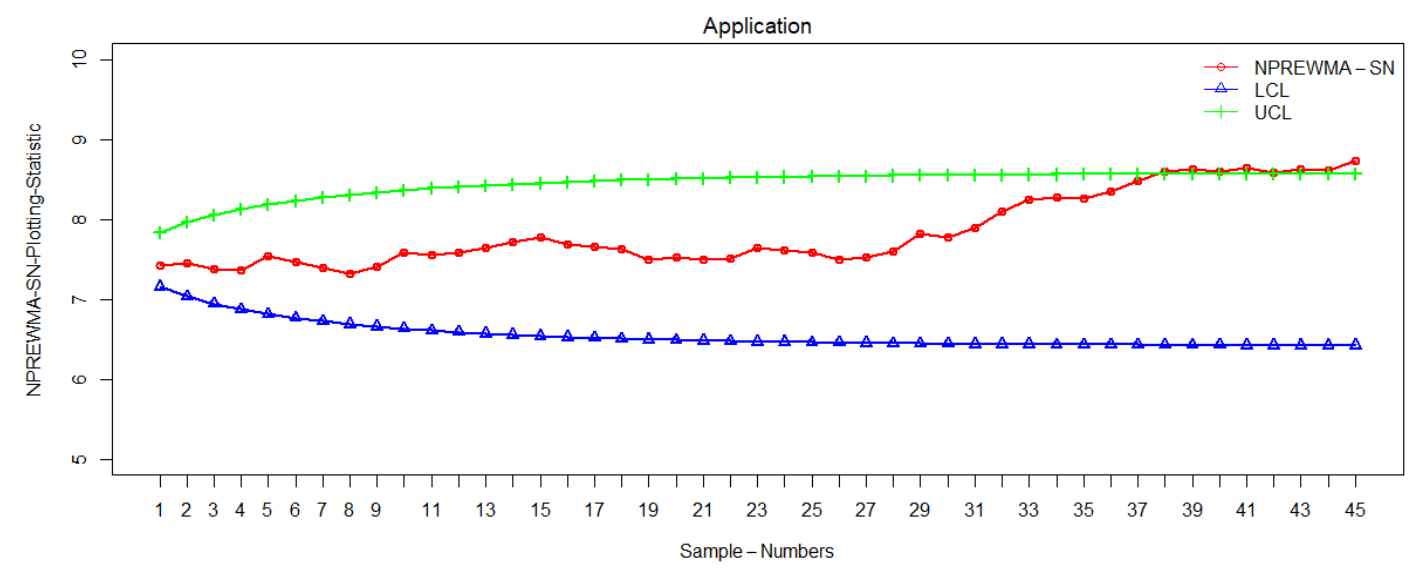

Figure 7. Real-life application of the proposed chart in a hard-bake manufacturing process.

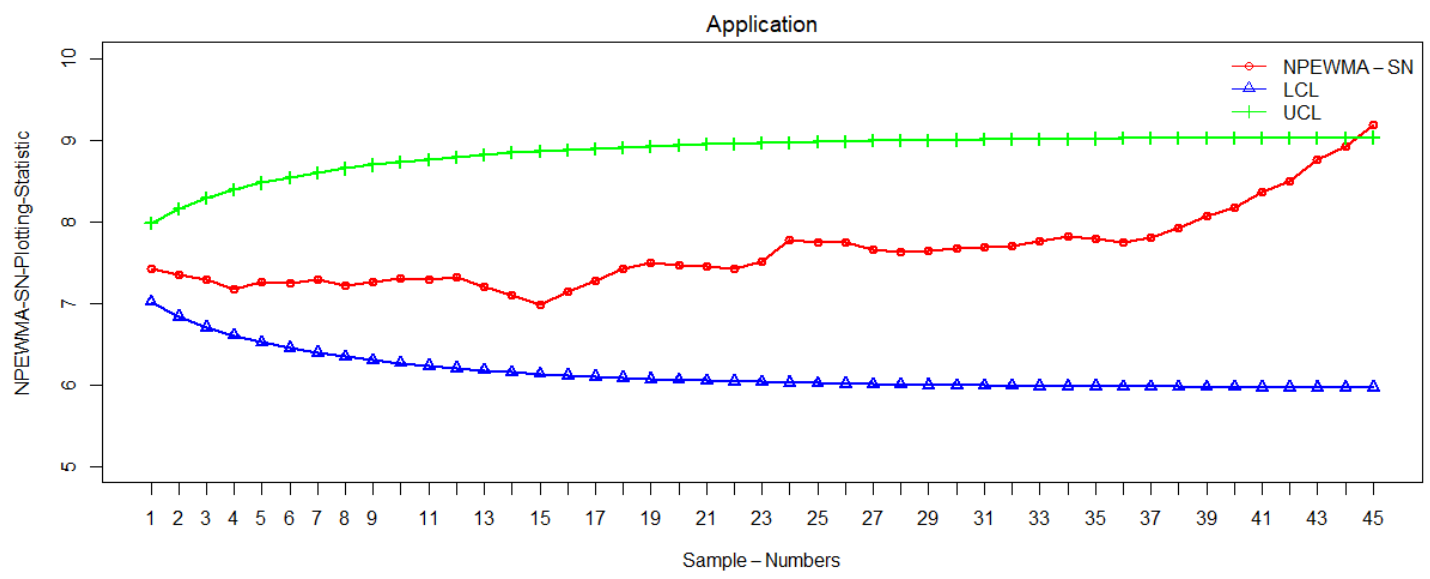

Figure 8. Real-life application of the NPEWMA-SN control chart in a hard-bake manufacturing process. 


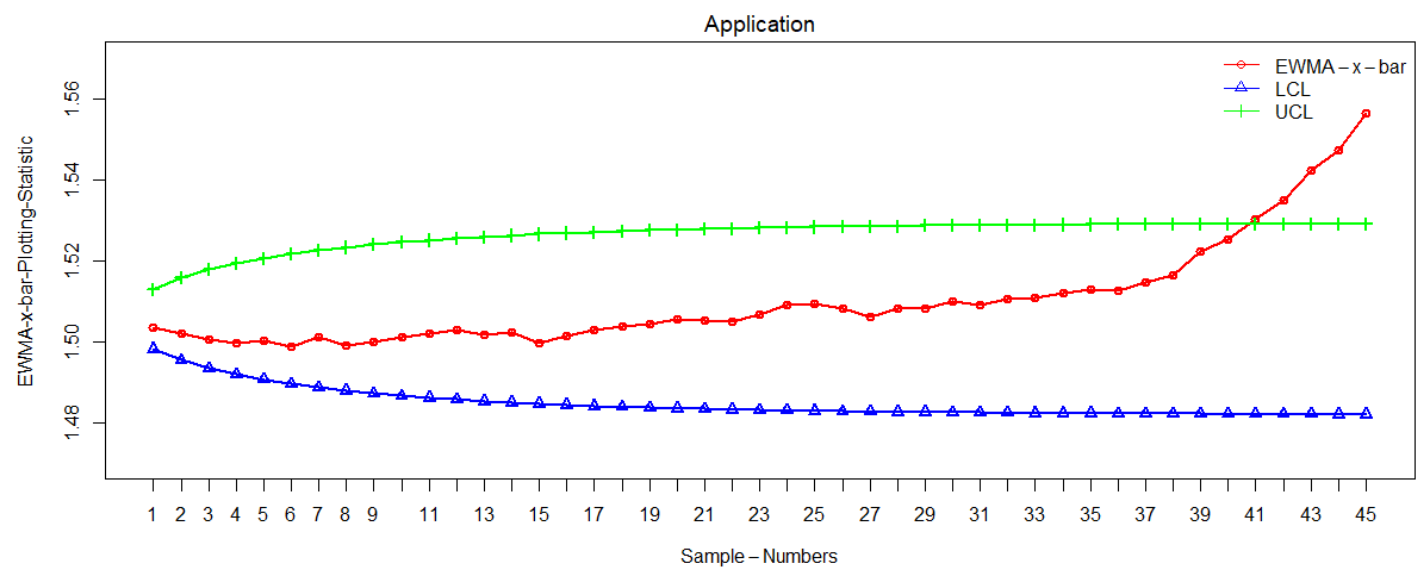

Figure 9. Real-life application of the EWMA-X control chart in a hard-bake manufacturing process.

\section{Summary and Conclusions}

SPC tools are used to detect aberrant changes in the process parameters, and the control chart is one of them. Control charts are designed under the normality assumptions of the process, but in many situations, normality assumption does not meet or process distribution is unknown. In such situations, the need for distribution-free control charts arises, as these charts are IC robust for all the continuous distributions. To make the charting structure more efficient, different extensions and modifications have been made in the literature, and the RSS scheme is one of them. In this study, a new NP monitoring scheme namely, NPREWMA-SN, based on a sign statistic under an RSS scheme has been proposed for monitoring small and persistent shifts in the location parameter. It is observed that the proposed chart provides a sensitive design structure for efficient process monitoring. Moreover, the RSS scheme provides an unbiased, efficient, and inexpensive estimate of the process parameter(s). The comparisons of the proposed method with NPEWMA-SN and classical EWMA- $\bar{X}$ schemes advocate its dominance over its competitors. An illustrative example on the substrate manufacturing process is also provided in support of the proposed study. It concludes that the proposed scheme has performed better toward small and persistent shifts detection at small values of smoothing parameters. The OOC performance of the proposed chart in Laplace (sharped peak) and logistic distributions is far better. The OOC performance of the proposed chart under the normal, heavy-tailed ' $t$ ' and contaminated processes is comparatively less effective. However, the proposed chart at small and moderate shifts in the process location performs uniformly better than existing counterparts under all the process distributions incorporated in this study. This work can be extended for estimation of the process parameters, multivariate scenarios, dynamic profiling analysis, etc.

Author Contributions: Conceptualization: S.A. and Z.A. Methodology: S.A. and Z.A. Software: S.A. and Z.A. Supervision: X.Z., Y.L. Visualization: H.Z.N., M.R. Writing original draft: S.A. Writing-review and editing: Z.A., H.Z.N. and M.R. All authors have read and agreed to the published version of the manuscript.

Funding: The aforementioned work was partially supported by National Natural Science Foundation of China No. 11731015 Guangzhou university research funding 69-6209254, 220030401 (Xingfa Zhang), National Natural Science Foundation of China Nos. 11731015, 11571148 (Yuan Li).

Acknowledgments: The aforementioned work was partially supported by National Natural Science Foundation of China (Nos. 11731015 and 11571148). The authors are grateful to the editor and referees for their constructive comments that led to substantial improvements in the article.

Conflicts of Interest: The author(s) declare no potential conflicts of interest with respect to the research, authorship and/or publication of this article.

\section{References}

1. Montgomery, D.C. Introduction to Statistical Quality Control, 7th ed.; John Wiley \& Sons: New York, NY, USA, 2012. 
2. Shewhart, W.A. Some applications of statistical methods to the analysis of physical and engineering data. Bell Syst. Tech. J. 1924, 3, 43-87. [CrossRef]

3. Qiu, P.; Li, Z. On nonparametric statistical process control of univariate processes. Technometrics 2011, 53, 390-405. [CrossRef]

4. Chakraborti, S.; Graham, M. Nonparametric (distribution-free) control charts: An updated overview and some results. Qual. Eng. 2019, 31, 1-22. [CrossRef]

5. Amin, R.W.; Reynolds, M.R., Jr.; Saad, B. Nonparametric quality control charts based on the sign statistic. Commun. Stat. Theory Methods 1995, 24, 1597-1623. [CrossRef]

6. Bakir, S.T. A distribution-free Shewhart quality control chart based on signed-ranks. Qual. Eng. 2004, 16, 613-623. [CrossRef]

7. Chakraborti, S.; Van der Laan, P.; Van de Wiel, M. A class of distribution-free control charts. J. R. Stat. Soc. Ser. C (Appl. Stat. ) 2004, 53, 443-462. [CrossRef]

8. Chakraborti, S.; Van de Wiel, M.A. A Nonparametric Control Chart Based on the Mann-Whitney Statistic; Institute of Mathematical Statistics: Beachwood, OH, USA, 2008.

9. Chakraborti, S.; Eryilmaz, S.; Human, S.W. A phase II nonparametric control chart based on precedence statistics with runs-type signaling rules. Comput. Stat. Data Anal. 2009, 53, 1054-1065. [CrossRef]

10. Bakir, S.T.; Reynolds, M.R. A nonparametric procedure for process control based on within-group ranking. Technometrics 1979, 21, 175-183. [CrossRef]

11. Mukherjee, A.; Graham, M.A.; Chakraborti, S. Distribution-free exceedance CUSUM control charts for location. Commun. Stat. Simul. Comput. 2013, 42, 1153-1187. [CrossRef]

12. Koutras, M.V.; Triantafyllou, I.S. Recent Advances on Univariate Distribution-Free Shewhart-Type Control Charts. In Distribution-Free Methods for Statistical Process Monitoring and Control; Springer: Berlin/Heidelberg, Germany, 2020. [CrossRef]

13. Yang, S.-F.; Lin, J.-S.; Cheng, S.W. A new nonparametric EWMA sign control chart. Expert Syst. Appl. 2011, 38, 6239-6243. [CrossRef]

14. Graham, M.A.; Chakraborti, S.; Human, S.W. A nonparametric exponentially weighted moving average signed-rank chart for monitoring location. Comput. Stat. Data Anal. 2011, 55, 2490-2503. [CrossRef]

15. Haq, A. A new nonparametric synthetic EWMA control chart for monitoring process mean. Commun. Stat. Simul. Comput. 2019, 48, 1665-1676. [CrossRef]

16. Haq, A. A nonparametric EWMA chart with auxiliary information for process mean. Commun. Stat. Theory Methods 2020, 49, 1232-1247. [CrossRef]

17. Abbas, Z.; Nazir, H.Z.; Abid, M.; Akhtar, N.; Riaz, M. Enhanced nonparametric control charts under simple and ranked set sampling schemes. Trans. Inst. Meas. Control 2020. [CrossRef]

18. McIntyre, G. A method for unbiased selective sampling, using ranked sets. Aust. J. Agric. Res. 1952, 3, 385-390. [CrossRef]

19. Song, Z.; Mukherjee, A.; Zhang, J. An efficient approach of designing distribution-free exponentially weighted moving average schemes with dynamic fast initial response for joint monitoring of location and scale. J. Stat. Comput. Simul. 2020. [CrossRef]

20. Abbasi, S.A. Efficient Control Charts for Monitoring Process CV Using Auxiliary Information. IEEE Access 2020, 8, 46176-46192. [CrossRef]

21. Abbasi, S.A.; Riaz, M.; Ahmad, S.; Sanusi, R.A.; Abid, M. New efficient exponentially weighted moving average variability charts based on auxiliary information. Qual. Reliab. Eng. Int. 2020. [CrossRef]

22. Abid, M.; Nazir, H.Z.; Riaz, M.; Lin, Z. Use of ranked set sampling in nonparametric control charts. J. Chin. Inst. Eng. 2016, 39, 627-636. [CrossRef]

23. Abid, M.; Nazir, H.Z.; Riaz, M.; Lin, Z. An Efficient Nonparametric EWMA Wilcoxon Signed-Rank Chart for Monitoring Location. Qual. Reliab. Eng. Int. 2017, 33, 669-685. [CrossRef]

24. Abid, M.; Nazir, H.Z.; Riaz, M.; Lin, Z. Investigating the impact of ranked set sampling in nonparametric CUSUM control charts. Qual. Reliab. Eng. Int. 2017, 33, 203-214. [CrossRef]

25. Abbasi, S.A.; Abbas, T.; Adegoke, N.A. Efficient CV Control Charts Based on Ranked Set Sampling. IEEE Access 2019, 7, 78050-78062. [CrossRef]

26. Graham, M.; Human, S.W.; Chakraborti, S. A Nonparametric EWMA Control Chart Based on the Sign Statistic; Faculty of Agricultural Sciences, University of Pretoria: Pretoria, South Africa, 2009. 
27. Hussain, S.; Mei, S.; Riaz, M.; Abbasi, S.A. On Phase-I Monitoring of Process Location Parameter with Auxiliary Information-Based Median Control Charts. Mathematics 2020. [CrossRef]

28. Raji, I.A.; Lee, M.H.; Riaz, M.; Abujiya, M.a.R.; Abbas, N. Outliers Detection Models in Shewhart Control Charts; an Application in Photolithography: A Semiconductor Manufacturing Industry. Mathematics 2020. [CrossRef]

29. Graham, M.A.; Chakraborti, S.; Human, S.W. A nonparametric EWMA sign chart for location based on individual measurements. Qual. Eng. 2011, 23, 227-241. [CrossRef]

30. Hettmansperger, T.P. The ranked-set sample sign test. J. Nonparametr. Stat. 1995, 4, 263-270. [CrossRef]

(C) 2020 by the authors. Licensee MDPI, Basel, Switzerland. This article is an open access article distributed under the terms and conditions of the Creative Commons Attribution (CC BY) license (http://creativecommons.org/licenses/by/4.0/). 